\title{
A Novel Numerical Procedure for Simulating Steady MHD Convective Flows of Radiative Casson Fluids over a Horizontal Stretching Sheet with Irregular Geometry under the Combined Influence of Temperature-Dependent Viscosity and Thermal Conductivity
}

\author{
Abderrahim Wakif 10 \\ Hassan II University, Faculty of Sciences AïnChock, Laboratory of Mechanics, B. P. 5366 Mâarif, Casablanca, Morocco \\ Correspondence should be addressed to Abderrahim Wakif; wakif.abderrahim@gmail.com
}

Received 22 November 2019; Revised 2 April 2020; Accepted 2 April 2020; Published 5 May 2020

Academic Editor: Vassilios C. Loukopoulos

Copyright $\odot 2020$ Abderrahim Wakif. This is an open access article distributed under the Creative Commons Attribution License, which permits unrestricted use, distribution, and reproduction in any medium, provided the original work is properly cited.

A novel mathematical computing analysis for steady magnetohydrodynamic convective flows of radiative Casson fluids moving over a nonlinearly elongating elastic sheet with a nonuniform thickness is established successfully in this numerical exploration. Also, the significance of an externally applied magnetic field with space-dependent strength on the development of MHD convective flows of Casson viscoplastic fluids is evaluated thoroughly by including the momentous influence of linear thermal radiation along with the temperature-dependent viscosity and thermal conductivity effects. By combining the assumption of the low-inducing magnetic field with the boundary layer approximations, the governing partial differential equations monitoring the current flow model are transmuted accordingly into a set of nonlinear coupled ordinary differential equations by invoking appropriate similarity transformations. Moreover, these derived differential equations are resolved numerically by utilizing a new innovative GDQLLM algorithm integrating the local linearization technique with the generalized differential quadrature method. On the other hand, the behaviours of velocity and temperature fields are deliberated properly through various graphical illustrations and different sets of flow parameters. However, the accurate datasets generated for the skin friction coefficient and local Nusselt number are presented separately in tabular displays, whose physical insights are discussed comprehensively via the slope linear regression method (SLRM). As main results, it is demonstrated that the higher values of the Casson viscoplastic parameter reduce significantly the fluid velocity within the boundary layer region, while a partial reverse tendency is observed near the stretching sheet as long as the wall thickness parameter is increased. Besides the previously mentioned hydrodynamical features, it is also depicted that the thermal field throughout the medium is enhanced considerably with the elevating values of these parameters.

\section{Introduction}

Linear and nonlinear rheological features of complex fluidic mediums have currently attracted widespread interests and considerable physical insights in the scientific debates owing to their valuable applications and widespread benefits over the whole usual fluids. Additionally, the non-Newtonian fluids have been extensively investigated during the past few decades by many scientists to explore their distinguishing rheological properties and establish new realistic constitutive laws permitting detailed explanations for some anomalous experimental observations. Indeed, the dynamic of most fluids together with their diverse characteristics cannot be properly described by the classical Navier-Stokes equations because the most common fluids used in industries are of a non-Newtonian kind, whose Cauchy stress and strain tensors can be expressed mathematically by quasilinear or purely nonlinear relationships depending greatly on their 
rheological behaviours, such as shear-thinning, shearthickening, viscoelasticity, viscoplasticity, material memory, Weissenberg effect, yield stress, die-swelling, and relaxation and retardation times, which are not defined in the Newtonian model. For this reason, the non-Newtonian fluids are widely used in various branches of science and bioengineering, for instance in the modelling of biological structures, drilling muds, cement slurries, petroleum, coated sheets, polymer manufacturing, foodstuffs, lubrication fields, and biomedical flows. Generally, the rheological attitudes of non-Newtonian fluids are dissimilar from those of viscous fluids. Besides, the transport equations governing the dynamical evolution of non-Newtonian fluids are fully nonlinear and highly complicated from the handling point of view compared with the Newtonian fluids. Furthermore, an analysis of the literature survey shows that there is no single constitutive equation that reveals all properties of such fluids. So, several constitutive non-Newtonian models have been suggested by the scientific communities. These models are principally classified into the differential, integral, and rate-type categories. In the rate-type rheological fluid models (e.g., Maxwell, Oldroyd-B, Jeffery, and Burgers models), the shear stress is an implicit relationship of the velocity gradient and its higher temporal derivatives. However, the differential rheological model (e.g., Leonov model) has been established mathematically to explain the existence of a set of discrete relaxation times. This phenomenological model is based on the presence of an internal thermodynamic equilibrium state, where the equilibrium elastic deformations have been memorized and any aberration kind from this state can cause a new nonequilibrium state. In contrast to the aforesaid models, the extra stress tensor for the integral constitutive model (e.g., K-BKZ and Wagner models) is expressed mathematically as the temporal integral of the memory function and the elastic potential distributions as well as the Cauchy-Green tensor and its inverse (i.e., Finger tensor). Moreover, this model shows an excellent capability of giving comparable upshots against the experimental rheological results for long dies towards the extrudate swell occurrence witnessed in some viscoelastic materials, such as acrylonitrile-butadiene-styrene nanocomposites and other polymer melts. This rheological phenomenon is due mainly to the elastic recovery of deformation, which reflects the relaxation effect on the material after leaving from the die state.

Numerous constitutive models have been proposed by pioneering researchers to describe complex convective boundary layer flows. For instance, Bilal et al. [1] adopted the tangent hyperbolic rheological model to examine the characteristics of an unsteady MHD convective flow of a non-Newtonian fluid moving over a nonlinear stretching porous sheet under the combined influence of convective heating and time-dependent magnetic field in the presence of magnetized dusty particles, heat generation/absorption, and variable thermal conductivity. Similarly, Bibi et al. [2] accomplished comprehensive computational estimations with the aid of the shooting technique along with the built-in MATLAB bvp4c package to handle approximatively an unsteady MHD convective flow of a tangent hyperbolic fluid conveying tiny magnetized particles over a linear stretching permeable sheet characterized by a uniform suction/injection velocity and affected by the significant effects of variable thermal conductivity, internal heat source/sink, convective heating, and time-dependent magnetic field. Otherwise, Mohebbi et al. [3] utilized the power-law model to evaluate the shear-thinning and shear-thickening features of a nonNewtonian fluid by conducting a numerical investigation on a fully developed forced heat transfer convection for a laminar non-Newtonian flow confined between two parallel plates containing partially porous media in the presence of a uniform internal arrangement of circular obstacles. In the same way, Liu et al. [4] developed an approximate approach to scrutinize the non-Newtonian rheological behaviour of fresh concrete based on the Herschel-Bulkley model [5] and various available experimental data sources. Sequel to the aforesaid background, Zhu et al. [6] investigated numerically the mutual influence of fluid yield stress and finite-size particles on a turbulent flow of a Bingham fluid [7] flowing inside a vertical channel under the effect of no-slip velocity boundary condition and the periodicity physical constraints in the streamwise and spanwise Cartesian directions. Based on the Carreau-Yasuda rheological model and Buongiorno's nonhomogeneous nanofluid model, Waqas et al. [8] proposed a realistic physical model to analyze the impacts of second-order velocity slip, zero nanoparticles mass flux condition, thermal radiation, and chemical reaction on the features of MHD convective flows over a convectively heated stretching surface in a porous medium by considering the coexistence of gyrotactic motile microorganisms and tiny solid particles and taken into account the thermophoresis and Brownian motion aspects. Keeping in mind the nonsimilarity characteristic of some flow problems causing by the non-Newtonian behaviour of the flowing fluids, Amanulla et al. [9] carried out an accurate numerical implementation to find nonsimilar solutions for the momentum and thermal boundary layers developed in a non-Darcy porous medium during the steady two-dimensional MHD free convection flow of a Prandtl-Eyring fluid past over an isothermal sphere under the effective influences of buoyancy forces and various slip conditions. Earlier, Muhammad et al. [10] exploited Buongiorno's mathematical formulation along with the generalized Fourier-Fick's law and Rosseland's approximation to establish a theoretical nanofluid model allowing to evaluate more efficiently the CattaneoChristov heat transfer for a three-dimensional MHDEMHD convective flow of an Eyring-Powell nanofluid flowing horizontally over an elongated Riga plate by considering the effects of nonlinear thermal radiation, activation energy, velocity slip condition, and convective heating. To explore the impact of shear-thinning and shear-thickening rheological properties on different kinds of transportation phenomena in non-Newtonian nanofluids under the combined effect of Brownian motion and thermophoresis diffusion of solid nanomaterials, Hayat et al. [11] extended the Sisko rheological fluid model [12] along with Buongiorno's mathematical formulation [13] and the vanishing mass flux condition for nanoparticles $[14,15]$ to simulate a threedimensional MHD forced convective nanofluid flow over a 
nonisothermal surface, which is taken to be materially impermeable, heated convectively, and stretched bidirectionally via linear velocities. In the objective of inspecting the surface drag forces in nanofluidic mediums, as well as the feasibility of enhancing their heat and mass transportations, Hayat et al. [16] addressed an imperative unsteady threedimensional MHD squeezing viscoelastic flow of a secondgrade nanofluid between two isothermal parallel disks, whose chemical species are controlled actively via Buongiorno's two-phase model under the assumption of small magnetic Reynolds number theory along with the blowing and suction effects. To foresee the impacts of shear-thinning and shear-thickening rheological properties, Sajid et al. [17] performed an approximative analytical investigation on the two-dimensional coating process for incompressible thirdgrade fluid flows in a thin slit located between a moving substrate and flexible/stiff coater by supposing that the coating layer thickness is greatly less than the blade length. Likewise, Arifuzzaman et al. [18] utilized the explicit finite difference technique to analyze the heat and mass transfer features of unsteady MHD radiative-convective flows of fourth-grade fluids moving perpendicularly from an isothermal permeable surface in a porous medium under the effects of magnetic field, suction/injection velocity, nonlinear order chemical reaction, heat generation, viscous dissipation, and buoyancy forces.

Due to the progress in the rheology of homogeneous and nonhomogeneous viscoelastic mixtures employed in widespread industrial and engineering areas, various constitutive rheological relationships have been developed for biological and polymeric rate-type liquids to reveal the memory and elasticity features of these liquids by introducing the relaxation and retardation times via the Oldroyd-B rheological model [19]. In the range of low shear rate model, the dynamic viscosity of Oldroyd-B fluids found to be increasingly varied, while this trend is reversed for higher shear rates indicating that these materials possess both solid and liquid characteristics. In particular, these properties can be constrained rheologically in some cases to deduce the Maxwell model [20] by disappearing the retardation time characterized by the solid tendency of viscoelastic fluids, in which the fluidic medium cannot respond instantaneously to any change in shear stress. In this direction, it is worth noting here that the Oldroyd-B model is incapable to explain the shear-thinning and shear-thickening rheological phenomena observed in some physiological and biological fluids (e.g., blood, serum, urine, saliva, and tears). Further, this model exhibits a quasilinear relationship between the shear stress and the shear strain rate, while this rheological dependency becomes a linear relationship for the Maxwell model. Among the most common rate-type model that having a time derivative in its linear rheological constitutive law and offering the possibility to investigate easily the viscoelastic behaviour of non-Newtonian fluids, we find the constitutive rheological Jeffrey's fluid model [21]. Bearing in mind the importance of this rheological model and its simplicity in simulating many realistic viscoelastic flows encountered widely in the bioscience sector and technology, this linear rheological law has lately obtained exceptional interest by researchers. In this regard, Hayat et al. [22] exploited the constitutive rheological equation of the Jeffrey model to examine the probable effects of an applied magnetic field, viscous dissipation, and internal heat source on the stagnation point flow of a non-Newtonian viscoelastic fluid towards a nonlinear stretching surface, which is characterized by a variable surface thickness and heated isothermally via an appropriate melting process. In another relevant investigation, Hayat et al. [23] inspected a three-dimensional MHD convective flow of an Oldroyd-B nanofluid moving horizontally over a bidirectionally stretching surface under the effects of zero nanoparticles mass flux condition, convective heating, Brownian motion, and thermophoresis. Because of the substantial interest given to these non-Newtonian models, Koteswara Reddy et al. [24] proposed an adjustable rheological model to analyze the features of MHD mixed convection viscoelastic flows of Maxwell/Jeffery/Oldroyd-B nanofluids moving vertically over a nonisothermal conical geometry by considering the existence of an internal heat source/sink along with the impact of thermophoresis and Brownian motion of solid nanoparticles. In a particular case, Awias et al. [25] carried out a numerical implementation via the bvp4c technique to study three-dimensional mixed convection flows of Maxwellian nanofluids driven by a vertically rotating and stretching isothermal cylinder, under the influences of various interactions including thermal/mass buoyancy, magnetic, thermophoretic, and Brownian forces. On the other hand, the existence of multiple relaxation times in the response of some materials (e.g., Earth's mantle and asphalt mastics) in creep and stress relaxation examinations can be adequately explained by another specified rate-type fluid model of higher order. For this purpose, many researchers prefer modelling numerous complex flow problems with the help of the Burgers rheological model [26] due to its capability of describing the coexistence of two different relaxation times. In the framework of this advanced rate-type fluid category, Hayat et al. [27] assumed the generalized Fourier-Fick's law along with Buongiorno's nanofluid model to analyze the heat and mass transportation phenomena occurred during the steady two-dimensional convective flows of Burgers nanofluids over a linearly stretching isothermal sheet.

Later on, various experimental investigations indicated that the Casson rheological fluid model [28] is still the best rheological way to understand the non-Newtonian behaviour of many viscoplastic fluids, like chocolate, blood, tomato sauce, paint, shampoo syrup, some polymer solutions, and other colloidal suspensions. This kind of fluids exhibits a shear-thinning rheological behaviour (i.e., pseudoplastic feature), whose viscosity decreases with the increasing shearing rate as long as the stress upsurges above a critical value recognized as the yield stress. More precisely, the presence of mineral ingredients in a liquid increases significantly the dynamic viscosity of mediums and modifies its rheological characteristic to a non-Newtonian behaviour with a rheological tendency to an increase in the fluidity, when the resulting mixture is sheared. However, the fluidic mediums show the capability to gel under certain situations, when it is not sheared. In this context, Kelessidis and Maglione [29] realized an exhaustive statistical analysis on 
the Casson and Robertson-Stiff rheological models [28, 30] via various existing experimental viscometric data generated especially for water-Greek bentonite suspensions to choose the excellent rheological category permitting a more realistic description of the studied aqueous medium. After successful attempts, they concluded that both suggested models can describe well the addressed sample data, in which the true shear rates for the Casson and Robertson-Stiff models are found to be higher compared with the Newtonian shear rates, whose differences become lesser at higher shear rates. These findings indicate an assessment in the Newtonian behaviour at greater shear rates. In the framework of the practical use of non-Newtonian Casson fluids, Qasim and Noreen [31] exploited the stability analysis theory to illustrate more evidently the possibility of generating multiple solutions for dissipative boundary layer flows of Casson fluids moving over a porous shrinking surface. More recently, Bhatti et al. [32] studied semianalytically MHD radiative Casson fluid flows from a nonisothermal linear stretching surface by including the Hall and ion-slip effects in the momentum equation via the generalized form of Ohm's law and imposing local convective heating as a feasible thermal condition at the horizontal boundary for the energy conservation equation.

Building upon the aforementioned literature review, the authors tackled their flow problems by utilizing various numerical and semianalytical procedures, such as Runge-Kutta method (RKM), spectral local linearization method (SLLM), spectral quasilinearization method (SQLM), spectral relaxation method (SRM), Keller box method (KBM), homotopy perturbation method (HPM), and homotopy analysis method (HAM), which are extensively employed in other related investigations. Besides, the analysis of these previous research works reveals that there is a greater lack of innovative research studies on the numerical analysis topic dealing realistically with the heat transportation phenomenon via non-Newtonian flows over complex geometries (e.g., irregular sheet, needle, cone, sphere, and cylinder). Motivating by these facts, a new numerical method is established in this scrutiny to explore the rheological fluid flow and heat transfer characteristics of radiative Casson viscoplastic fluids having temperaturedependent thermophysical properties (i.e., dynamic viscosity and thermal conductivity), surrounding by a nonuniform applied magnetic field and moving horizontally over an isothermal stretching sheet with variable thickness. Moreover, the graphical and tabular highlights produced for every physical quantity showing up in the present numerical assessment (i.e., velocity, temperature, skin friction coefficient, and heat transfer rate) are discussed physically through plausible explanations. Furthermore, the flow patterns are further displayed for velocity boundary layers and streamlines to inspect the consequence of applying a nonuniform magnetic field on the fluid flow properties.

\section{Problem Statement}

Let us consider a steady two-dimensional MHD convective flow of a Casson viscoplastic fluid exhibiting a temperature dependency for the dynamic viscosity and thermal conductivity and moving over a nonlinearly slender sheet of variable thickness $\varepsilon(x)$, which is heated uniformly via an imposed temperature $T_{w}$ and stretched horizontally in the positive sense of the $x$-axis with a spatially variable velocity $u_{x}(x)$ under the impacts of thermal radiation and variable magnetic field $\mathbf{B}=B(x) \mathbf{e}_{\mathbf{y}}$, where $\varepsilon(x)=2 c(x+a)^{(1-n) / 2}$, $u_{x}(x)=b(x+a)^{n}$, and $B(x)=B_{0}(x+a)^{(n-1) / 2}$. Here, the dimensional constants $(a, b, c)$ are utilized in the expressions of $\varepsilon(x)$ and $u_{x}(x)$ to characterize the slender sheet geometry and its dynamical behaviour, while the exponent $n$ designates the velocity power parameter. Also, the constant $c$ is selected properly in this physical problem, in such a way that the sheet is sufficiently thin compared to its width $\varepsilon(x)$. As schematically portrayed in Figure 1, the mathematical characteristics of the studied flow geometry are presented in a Cartesian coordinate system $(x, y, z)$, whose unit vectors are $\left(\mathbf{e}_{\mathbf{x}}, \mathbf{e}_{\mathbf{y}}, \mathbf{e}_{\mathbf{z}}\right)$. In this configuration, the origin $O$ is located at the leading edge of the transverse section centre of the sheet, in which the $x$-axis is positioned horizontally along the symmetrical axis of the elastic sheet, while the $y$-axis is taken perpendicularly to the flow direction. As assumptions, the radiative fluid flow is supposed laminar, incompressible, and having a weaker electrical conductivity $\sigma$ and can be developed with a low Reynolds magnetic number, in which the induced magnetic field can be neglected reasonably.

By adopting the linearized form of the Rosseland radiative heat flux model [33] along with the boundary layer approximations, the various transport equations (i.e., mass, momentum, and energy conservation equations) governing the present flow problem are stated as follows:

$$
\begin{aligned}
\frac{\partial u}{\partial x}+\frac{\partial v}{\partial y}= & 0 \\
\rho\left(u \frac{\partial u}{\partial x}+v \frac{\partial u}{\partial y}\right)= & \left(1+\frac{1}{\beta}\right) \mu_{T}\left(\frac{\partial^{2} u}{\partial y^{2}}\right)+\left(1+\frac{1}{\beta}\right)\left(\frac{\partial \mu_{T}}{\partial T}\right) \\
& \cdot\left(\frac{\partial T}{\partial y}\right)\left(\frac{\partial u}{\partial y}\right)-\sigma B^{2}(x) u
\end{aligned}
$$

$$
\begin{aligned}
\left(\rho C_{P}\right)\left(u \frac{\partial T}{\partial x}+v \frac{\partial T}{\partial y}\right)= & k_{T}\left(\frac{\partial^{2} T}{\partial y^{2}}\right)+\left(\frac{\partial k_{T}}{\partial T}\right)\left(\frac{\partial T}{\partial y}\right)^{2} \\
& +\frac{16 \sigma_{e} T_{\infty}^{3}}{3 \beta_{R}}\left(\frac{\partial^{2} T}{\partial y^{2}}\right)
\end{aligned}
$$

As emphasized above, $u$ and $v$ represent the velocity components in the $x$ and $y$ directions, respectively, $\rho$ means the fluid density, $C_{P}$ corresponds to the specific heat of the Casson fluid, $\beta$ indicates the Casson fluid parameter, $\mu_{T}$ refers to the plastic dynamic viscosity of the medium, $T$ symbolizes the fluid temperature, $k_{T}$ specifies the thermal conductivity of the fluid, $\sigma_{e}$ signifies the Stefan-Boltzmann constant, $\beta_{R}$ designates the mean absorption coefficient, $\sigma$ defines the electrical conductivity of the medium, and $B(x)$ shows the strength of the variable magnetic field. 

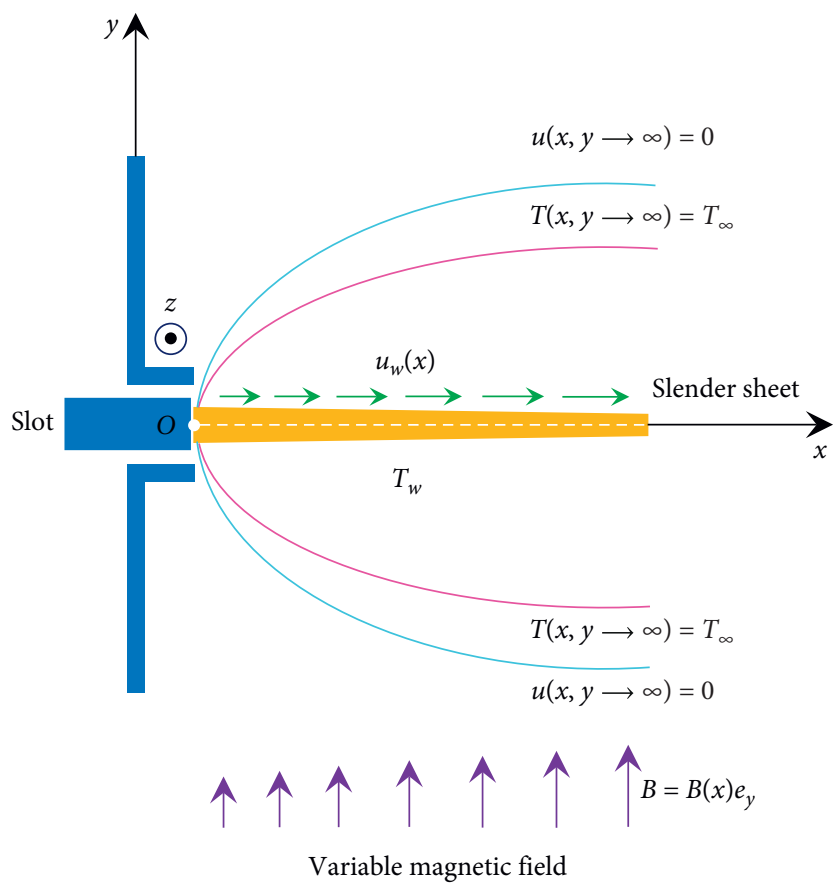

- Casson fluid velocity boundary layer

_ : Casson fluid temperature boundary layer

FIgURE 1: Schematic diagram of the present flow problem.

By assuming a linear temperature dependency for the thermophysical quantities $\left(\mu_{T}, k_{T}\right)$, we get [34]

$$
\begin{aligned}
& \mu_{T}=\mu\left(1-\Lambda_{\mu}\left(\frac{T-T_{\infty}}{T_{w}-T_{\infty}}\right)\right), \\
& k_{T}=k\left(1+\Lambda_{k}\left(\frac{T-T_{\infty}}{T_{w}-T_{\infty}}\right)\right) .
\end{aligned}
$$

Moreover, the thermophysical properties $(\mu, k)$ shown above designate the effective values of the variable quantities $\left(\mu_{T}, k_{T}\right)$ at the stream temperature $T_{\infty}$, whereas dimensionless quantities $\Lambda_{\mu}$ and $\Lambda_{k}$ represent the variable viscosity and thermal conductivity parameters, correspondingly.

For the flow geometry under consideration, the prescribed boundary conditions regulating equations (1)-(3) are written as follows:

$$
\begin{aligned}
u(x, y) & =u_{w}(x)=b(x+a)^{n}, \\
v(x, y) & =0, \\
T(x, y) & =T_{w}, \\
\text { at } y & =\frac{\varepsilon(x)}{2}, \\
u(x, y) & \longrightarrow 0, \\
T(x, y) & \longrightarrow T_{\infty}, \\
\text { as } y & \longrightarrow \infty .
\end{aligned}
$$

Accordingly, equations (1)-(3) can be nondimensionalized by invoking the following authentic similarity transformations:

$$
\begin{aligned}
u(x, y)= & b(x+a)^{n} F^{\prime}(\zeta), \\
v(x, y)= & -\left(\frac{(n+1) v b(x+a)^{(n-1)}}{2}\right)^{1 / 2} \\
& \cdot\left(F(\zeta)+\left(\frac{n-1}{n+1}\right) \zeta F^{\prime}(\zeta)\right), \\
\zeta= & \left(\frac{(n+1) b(x+a)^{(n-1)}}{2 v}\right)^{1 / 2} y, \\
\psi(x, y)= & \left(\frac{2 v b(x+a)^{(n+1)}}{(n+1)}\right)^{1 / 2} F(\zeta), \\
\Theta(\zeta)= & \frac{T(x, y)-T_{\infty}}{T_{w}-T_{\infty}} .
\end{aligned}
$$

In this state, the resulting nonlinear ordinary differential equations (ODEs) with their proper boundary conditions are given by

$$
\begin{gathered}
\left(1+\frac{1}{\beta}\right) F^{\prime \prime \prime}-\frac{2 M}{(n+1)} F^{\prime}+F F^{\prime \prime}-\frac{2 n}{(n+1)} F^{\prime 2} \\
-\left(1+\frac{1}{\beta}\right) \Lambda_{\mu} F^{\prime \prime \prime} \Theta-\left(1+\frac{1}{\beta}\right) \Lambda_{\mu} F^{\prime \prime} \Theta^{\prime}=0
\end{gathered}
$$




$$
\begin{aligned}
\left(1+R_{D}\right) \Theta^{\prime \prime}+ & \operatorname{Pr} F \Theta^{\prime}+\Lambda_{k} \Theta \Theta^{\prime \prime}+\Lambda_{k} \Theta^{\prime 2}=0, \\
F(\zeta) & =\left(\frac{1-n}{1+n}\right) \chi, \\
F^{\prime}(\zeta) & =1, \\
\Theta(\zeta) & =1, \\
\text { at } \zeta & =\chi, \\
F^{\prime}(\zeta) & \longrightarrow 0, \\
\Theta(\zeta) & \longrightarrow 0, \\
\text { as } \zeta & \longrightarrow \infty .
\end{aligned}
$$

As underlined above, the prime superscripts designate the derivation with respect to $\zeta$. Also, the physical parameters $M, R_{D}$, Pr, and $\chi$ involved in equations (8)-(10) are defined formally as follows:

$$
\begin{aligned}
M & =\frac{\sigma B_{0}^{2}}{b \rho} \text { (magnetic parameter), } \\
R_{D} & =\frac{16 \sigma_{e} T_{\infty}^{3}}{3 \beta_{R} k} \text { (radiation parameter), } \\
\operatorname{Pr} & =\frac{\left(\rho C_{P}\right) v}{k}(\text { Prandtl number), } \\
\chi & =c\left(\frac{b n+b}{2 v}\right)^{(1 / 2)} \text { (wall thickness parameter). }
\end{aligned}
$$

For further simplifications, we set the following feasible changes:

$$
\left\{\begin{array}{l}
\zeta=\eta+\chi \\
F(\zeta)=F(\eta+\chi)=f(\eta) \\
\Theta(\zeta)=\Theta(\eta+\chi)=\theta(\eta)
\end{array}\right.
$$

Therefore, the nonlinear differential system described by equations (8)-(11) is altered to

$$
\begin{aligned}
&\left(1+\frac{1}{\beta}\right) f^{\prime \prime \prime}-\frac{2 M}{(n+1)} f^{\prime}+f f^{\prime \prime}-\frac{2 n}{(n+1)} f^{\prime 2} \\
&-\left(1+\frac{1}{\beta}\right) \Lambda_{\mu} f^{\prime \prime \prime} \theta-\left(1+\frac{1}{\beta}\right) \Lambda_{\mu} f^{\prime \prime} \theta^{\prime}=0, \\
&\left(1+R_{D}\right) \theta^{\prime \prime}+\operatorname{Pr} f \theta^{\prime}+\Lambda_{k} \theta \theta^{\prime \prime}+\Lambda_{k} \theta^{\prime 2}=0, \\
& f(\eta)=\left(\frac{1-n}{1+n}\right) \chi, \\
& f^{\prime}(\eta)=1, \\
& \theta(\eta)=1, \\
& \text { at } \eta=0,
\end{aligned}
$$

$$
\begin{gathered}
f^{\prime}(\eta) \longrightarrow 0, \\
\theta(\eta) \longrightarrow 0, \\
\text { as } \eta \longrightarrow \infty .
\end{gathered}
$$

Here, the prime notations are utilized to refer to the derivation for the variable $\eta$.

\section{Engineering Quantities of Interest}

In this physical problem, the significance of the principal engineering quantities of major importance can be scrutinized appropriately by estimating locally the magnitude of dragging forces and heat transfer rate at the stretching surface in terms of the skin friction coefficient $C_{f x}$ and Nusselt number $N u_{x}$. These physical quantities are defined as follows:

$$
\begin{aligned}
C_{f x} & =\frac{2 \tau_{x y}(x, y=\varepsilon(x) / 2)}{\rho_{f} u_{w}^{2}(x)}, \\
N u_{x} & =\frac{(x+a) q_{T}}{k\left(T_{w}-T_{\infty}\right)},
\end{aligned}
$$

in which

$$
\begin{aligned}
\tau_{x y}\left(x, y=\frac{\varepsilon(x)}{2}\right) & =\mu_{T}\left(1+\frac{1}{\beta}\right)\left(\left(\frac{\partial u}{\partial y}\right)+\left(\frac{\partial v}{\partial x}\right)\right)_{y=(\varepsilon(x) / 2)}, \\
q_{T} & =-\left(k_{T}+\frac{16 \sigma_{e} T_{\infty}^{3}}{3 \beta_{R}}\right)\left(\frac{\partial T}{\partial y}\right)_{y=(\varepsilon(x) / 2)} .
\end{aligned}
$$

By making use of equations (5), (7), and (13), we get the following expressions:

$$
\begin{aligned}
C_{f r} & =\frac{(2 n+2)^{(1 / 2)}}{\beta(\beta+1)^{-1}}\left(\Lambda_{\mu} \theta(0)-1\right) f^{\prime \prime}(0), \\
N u_{r} & =-\left(\frac{n+1}{2}\right)^{(1 / 2)}\left(\Lambda_{k} \theta(0)+\left(1+R_{D}\right)\right) \theta^{\prime}(0) .
\end{aligned}
$$

As mentioned above, the physical quantities $C_{f r}$ and $N u_{r}$ represent the reduced forms of the local skin friction coefficient $C_{f x}$ and Nusselt number $N u_{x}$, which are given by

$$
\begin{gathered}
C_{f r}=-\operatorname{Re}_{x}^{1 / 2} C_{f x}, \\
N u_{r}=\operatorname{Re}_{x}^{-1 / 2} N u_{x} .
\end{gathered}
$$

Here, $\operatorname{Re}_{x}$ denotes the local Reynolds number, where $\operatorname{Re}_{x}=\left((x+a) u_{w}(x) / v\right)$.

\section{Numerical Modeling Strategy}

A new efficient numerical procedure is presented exclusively in this investigation to solve the nonlinear differential system described in the fluid motion and heat transfer features of a 
non-Newtonian viscoplastic fluid possessing temperaturedependent viscosity and thermal conductivity and flowing over an isothermal stretching sheet with irregular and axisymmetric geometry under the influence of a nonuniform applied magnetic field with the presence of thermal radiation. The proposed numerical algorithm is built on a purely theoretical framework. In this regard, the resulting nonlinear differential system of equations (14)-(17) can be linearized and decoupled accordingly to reduce them into a sequence of dependent subsystems of linear ODEs. After several successful rearrangements, the constructed differential subsystems are discretized spatially to be tackled numerically by utilizing the generalized differential quadrature local linearization method (GDQLLM).

Based on the pioneering mathematical findings reported by Bellman and Kalaba [35], the present flow model can be handled iteratively by applying the local linearization technique (LLT) to the following generated system:

$$
\left(S_{N L}\right):\left\{\begin{array}{l}
\theta=1, \quad \text { when } \eta=0, \\
\left(1+R_{D}\right) \theta^{\prime \prime}+\operatorname{Pr} f \theta \prime+\Lambda_{k} \theta \theta^{\prime \prime}+\Lambda_{k} \theta^{2}=0, \quad \text { where } 0<\eta<\eta_{\infty}, \\
\theta=0, \quad \text { when } \eta=\eta_{\infty}, \\
\left(1+\frac{1}{\beta}\right) g^{\prime \prime}-\frac{2 M g}{n+1}+f g^{\prime}-\frac{2 n g^{2}}{n+1}-\left(1+\frac{1}{\beta}\right) \Lambda_{\mu} g^{\prime \prime} \theta-\left(1+\frac{1}{\beta}\right) \Lambda_{\mu} g^{\prime} \theta^{\prime}=0, \quad \text { when } \eta=0, \\
g=0, \quad \text { when } \eta=\eta_{\infty}, \\
f=\left(\frac{1-n}{1+n}\right) \chi, \quad \text { when } \eta=0, \\
f^{\prime}=g, \quad \text { where } 0<\eta<\eta_{\infty} .
\end{array}\right.
$$

As highlighted in equation (22), the value $\eta_{\infty}$ corresponds to the estimated dimensionless boundary layer thickness assuring the asymptotical achievement of the stream boundary conditions with higher precision. Consequently, the linearized form of the nonlinear differential system $\left(S_{N L}\right)$ is written as follows:

$\left(S_{L}\right)$ :

$$
\left\{\begin{array}{l}
\theta_{r+1}=1, \quad \text { when } \eta=0, \\
A_{1} \theta_{r+1}^{\prime \prime}+A_{2} \theta_{r+1}^{\prime}+A_{3} \theta_{r+1}=R_{1}, \quad \text { where } 0<\eta<\eta_{\infty}, \\
\theta_{r+1}=0, \quad \text { when } \eta=\eta_{\infty}, \\
g_{r+1}=1, \quad \text { when } \eta=0, \\
B_{1} g_{r+1}^{\prime \prime}+B_{2} g_{r+1}^{\prime}+B_{3} g_{r+1}=R_{2}, \quad \text { where } 0<\eta<\eta_{\infty}, \\
g_{r+1}=0, \quad \text { when } \eta=\eta_{\infty}, \\
f_{r+1}=\left(\frac{1-n}{1+n}\right) \chi, \quad \text { when } \eta=0, \\
f_{r+1}^{\prime}=R_{3}, \quad \text { where } 0<\eta<\eta_{\infty} .
\end{array}\right.
$$

Moreover, the solutions of the linear differential system $\left(S_{L}\right)$ can be adjusted iteratively through the functions $\left\{\left(A_{i}(\eta), B_{i}(\eta), R_{i}(\eta)\right) / 1 \leq i \leq 3\right.$ and $\left.0 \leq \eta \leq \eta_{\infty}\right\}$, which are expressed as follows:

$$
\begin{aligned}
& A_{1}(\eta)=\left(1+R_{D}\right)+\Lambda_{k} \theta_{r}(\eta), \\
& A_{2}(\eta)=\operatorname{Pr} f_{r}(\eta)+2 \Lambda_{k} \theta_{r}^{\prime}(\eta), \\
& A_{3}(\eta)=\Lambda_{k} \theta_{r}^{\prime \prime}(\eta), \\
& B_{1}(\eta)=\frac{\left(1-\Lambda_{\mu} \theta_{r+1}(\eta)\right)}{\beta(\beta+1)^{-1}} \\
& B_{2}(\eta)=f_{r}(\eta)-\frac{\Lambda_{\mu} \theta_{r+1}^{\prime}(\eta)}{\beta(\beta+1)^{-1}}, \\
& B_{3}(\eta)=-\left(\frac{2 M+4 n g_{r}(\eta)}{n+1}\right), \\
& R_{1}(\eta)=\Lambda_{k} \theta_{r}(\eta) \theta_{r}^{\prime \prime}(\eta)+\Lambda_{k} \theta_{r}^{\prime 2}(\eta), \\
& R_{2}(\eta)=-\frac{2 n g_{r}^{2}(\eta)}{n+1}, \\
& R_{3}(\eta)=g_{r+1}(\eta) .
\end{aligned}
$$


Here, the iterative subscripts $r$ and $(r+1)$ are utilized in equations (23)-(26) to indicate the previous and current estimations, respectively.

Furthermore, to boost the numerical procedure approved during the resolution steps, it is preferable to initialize the iterative process by testing solutions $\left\{\left(f_{0}(\eta), g_{0}(\eta), \theta_{0}(\eta)\right) / 0 \leq \eta \leq \eta_{\infty}\right\}$ verifying all the boundary conditions imposed on the differential system $\left(S_{L}\right)$. These initial guessed solutions take the following expressions:

$$
\begin{aligned}
& f_{0}(\eta)=1+\chi\left(\frac{1-n}{1+n}\right)-e^{-\eta} \\
& g_{0}(\eta)=e^{-\eta} \\
& \theta_{0}(\eta)=e^{-\eta}
\end{aligned}
$$

By applying the generalized differential quadrature method (GDQM) along with the modified Gauss-Lobatto collocation points [36-41], the resulting linearized system $\left(S_{L}\right)$ can be discretized spatially to give three decoupled linear algebraic subsystems $\left(S_{\theta}\right),\left(S_{g}\right)$, and $\left(S_{f}\right)$, whose unknowns are the approximate discrete values of the functions $\theta_{r+1}(\eta), g_{r+1}(\eta)$, and $f_{r+1}(\eta)$ in the computational domain $\left[0, \eta_{\infty}\right]$. These subsystems can be written in the following matrix forms:

$$
\begin{aligned}
& \left(S_{\theta}\right):\left\{\mathbf{A T}^{r+1}=\mathbf{R}_{1}, \quad \text { where } \mathbf{A}(1,:)=\mathbf{I}(1,:), \mathbf{A}(N,:)=\mathbf{I}(N,:), \mathbf{R}_{1}(1)=1 \text { and } \mathbf{R}_{1}(N)=0\right\}, \\
& \left(S_{g}\right):\left\{\mathbf{B G}^{r+1}=\mathbf{R}_{2}, \quad \text { where } \mathbf{B}(1,:)=\mathbf{I}(1,:), \mathbf{B}(N,:)=\mathbf{I}(N,:), \mathbf{R}_{2}(1)=1 \text { and } \mathbf{R}_{2}(N)=0\right\}, \\
& \left(S_{f}\right):\left\{\mathbf{C F}^{r+1}=\mathbf{R}_{3}, \quad \text { where } \mathbf{C}(1,:)=\mathbf{I}(1,:) \text { and } \mathbf{R}_{3}(1)=\left(\frac{1-n}{1+n}\right) \chi\right\} .
\end{aligned}
$$

Additionally, the square matrices $\{\mathbf{A}, \mathbf{B}, \mathbf{C}\}$ and vector columns $\left\{\mathbf{T}^{r+1}, \mathbf{G}^{r+1}, \mathbf{F}^{r+1}, \mathbf{R}_{1}, \mathbf{R}_{2}, \mathbf{R}_{3}\right\}$ are expressed as follows:

$$
\begin{aligned}
\mathbf{A} & =\operatorname{diag}\left[\left(A_{1}\left(\eta_{j}\right)\right)_{1 \leq j \leq N}\right] \mathbf{D}^{(2)}+\operatorname{diag}\left[\left(A_{2}\left(\eta_{j}\right)\right)_{1 \leq j \leq N}\right] \mathbf{D}^{(1)}+\operatorname{diag}\left[\left(A_{3}\left(\eta_{j}\right)\right)_{1 \leq j \leq N}\right] \mathbf{I}, \\
\mathbf{B} & =\operatorname{diag}\left[\left(B_{1}\left(\eta_{j}\right)\right)_{1 \leq j \leq N}\right] \mathbf{D}^{(2)}+\operatorname{diag}\left[\left(B_{2}\left(\eta_{j}\right)\right)_{1 \leq j \leq N}\right] \mathbf{D}^{(1)}+\operatorname{diag}\left[\left(B_{3}\left(\eta_{j}\right)\right)_{1 \leq j \leq N}\right] \mathbf{I}, \\
\mathbf{C} & =\mathbf{D}^{(1)}, \\
\mathbf{T}^{r+1} & =\left[\left(\theta_{r+1}\left(\eta_{j}\right)\right)_{1 \leq j \leq N}\right], \\
\mathbf{G}^{r+1} & =\left[\left(g_{r+1}\left(\eta_{j}\right)\right)_{1 \leq j \leq N}\right], \\
\mathbf{F}^{r+1} & =\left[\left(f_{r+1}\left(\eta_{j}\right)\right)_{1 \leq j \leq N}\right], \\
\mathbf{R}_{1} & =\left[\left(R_{1}\left(\eta_{j}\right)\right)_{1 \leq j \leq N}\right], \\
\mathbf{R}_{2} & =\left[\left(R_{2}\left(\eta_{j}\right)\right)_{1 \leq j \leq N}\right], \\
\mathbf{R}_{3} & =\left[\left(R_{3}\left(\eta_{j}\right)\right)_{1 \leq j \leq N}\right] .
\end{aligned}
$$


As shown above, $\quad \mathbf{D}^{(1)}=\left[\left(d_{i j}^{(1)}\right)_{1 \leq i, j \leq N}\right]$ and $\mathbf{D}^{(2)}=\left[\left(d_{i j}^{(2)}\right)_{1 \leq i, j \leq N}\right]$ symbolize the first- and second-order differentiation matrices, respectively, I denotes the unit matrix, the set $\left\{\eta_{j} / 1 \leq j \leq N\right\}$ highlights the modified

$$
\left\{\begin{array}{l}
d_{i j}^{(p)}=\frac{\prod_{k=1, k \neq i}^{N}\left(\tau_{i}-\tau_{k}\right)}{\eta_{\infty}^{p}\left(\tau_{i}-\tau_{j}\right) \prod_{k=1, k \neq i}^{N}\left(\tau_{i}-\tau_{k}\right)}, \\
d_{i j}^{(p)}=\frac{p}{\eta_{\infty}^{p}}\left(d_{i i}^{(p-1)} d_{i j}^{(1)}-\frac{d_{i j}^{(p-1)}}{\tau_{i}-\tau_{j}}\right), \\
d_{i j}^{(p)}=-\frac{1}{\eta_{\infty}^{p}} \sum_{k=1, k \neq i}^{N} d_{i k}^{(p)}, \\
\tau_{j}=\frac{\eta_{j}}{\eta_{\infty}}=\frac{1}{2}-\frac{1}{2} \cos \left(\frac{\pi j-\pi}{N-1}\right),
\end{array}\right.
$$

Furthermore, it bears noting that, after discretizing the differential system $\left(S_{L}\right)$, the derivative terms can be handled numerically in each collocation point $\eta_{i}$ as follows:

$$
\left\{\begin{array}{l}
f_{r+1}^{(p)}\left(\eta_{i}\right)=\sum_{j=1}^{N} d_{i j}^{(p)} f_{r+1}\left(\eta_{j}\right)=\mathbf{D}^{(p)}(i,:) \mathbf{F}_{r+1} \\
g_{r+1}^{(p)}\left(\eta_{i}\right)=\sum_{j=1}^{N} d_{i j}^{(p)} g_{r+1}\left(\eta_{j}\right)=\mathbf{D}^{(p)}(i,:) \mathbf{G}_{r+1}, \\
\theta_{r+1}^{(p)}\left(\eta_{i}\right)=\sum_{j=1}^{N} d_{i j}^{(p)} \theta_{r+1}\left(\eta_{j}\right)=\mathbf{D}^{(p)}(i,:) \mathbf{T}_{r+1}
\end{array}\right.
$$

where $p$ is the order of derivation concerning the variable $\eta$.

During the progression in the iterative procedure, the reduced quantities $C_{f r}$ and $N u_{r}$ are computed as follows:
Gauss-Lobatto collocation points, and $N$ represents the number of collocation nodal points $\eta_{j}$.

According to Shu [42], the components of the modified differentiation matrices $\mathbf{D}^{(1)}$ and $\mathbf{D}^{(2)}$ can be computed from the following general system:

for $p=1$ and $i \neq j$, where $1 \leq i, j \leq N$,

for $p \geq 2$ and $i \neq j$, where $1 \leq i, j \leq N$,

for $p \geq 1$ and $i=j$, where $1 \leq i, j \leq N$,

where $0 \leq \eta_{j} \leq \eta_{\infty}, 0 \leq \tau_{j} \leq 1$ and $1 \leq j \leq N$.

$$
\begin{aligned}
C_{f r} & =\frac{(2 n+2)^{(1 / 2)}}{\beta(\beta+1)^{-1}}\left(\Lambda_{\mu} T_{r+1}(1)-1\right) \mathbf{D}^{(1)}(1,:) \mathbf{G}_{r+1}, \\
N u_{r} & =-\left(\frac{n+1}{2}\right)^{(1 / 2)}\left(\Lambda_{k} T_{r+1}(1)+\left(1+R_{D}\right)\right) \mathbf{D}^{(1)}(1,:) \mathbf{T}_{r+1} .
\end{aligned}
$$

\section{Accuracy and Convergence of the Proposed Numerical Algorithm}

The convergence and constancy of the sequential iterative schemes established for the present boundary layer flow problem can be assessed fittingly from the nonlinear differential system $\left(S_{N L}\right)$ by considering the following residual functions:

$$
\begin{aligned}
& \operatorname{Res}_{g}(\eta)=\left\{\frac{g_{r+1}^{\prime \prime}(\eta)}{\beta(\beta+1)^{-1}}+f_{r+1}(\eta) g_{r+1}^{\prime}(\eta)-\frac{2 g_{r+1}(\eta)\left(M+n g_{r+1}(\eta)\right)}{n+1}-\frac{\Lambda_{\mu}\left(g_{r+1}^{\prime \prime}(\eta) \theta_{r+1}(\eta)+g_{r+1}^{\prime}(\eta) \theta_{r+1}^{\prime}(\eta)\right)}{\beta(\beta+1)^{-1}}\right\} \\
& \operatorname{Res}_{\theta}(\eta)=\left(1+R_{D}\right) \theta^{\prime \prime}(\eta)+\operatorname{Pr} f(\eta) \theta^{\prime}(\eta)+\Lambda_{k} \theta(\eta) \theta^{\prime \prime}(\eta)+\Lambda_{k} \theta^{\prime 2}(\eta) .
\end{aligned}
$$

After discretization, these residual functions take the following vector forms: 


$$
\operatorname{Res}_{\mathbf{g}}=\left\{\begin{array}{c}
\frac{\mathbf{D}^{(2)} \mathbf{G}_{r+1}}{\beta(\beta+1)^{-1}}+\operatorname{diag}\left(\left[\operatorname{diag}\left(\mathbf{F}_{r+1}\right)\right]\left[\operatorname{diag}\left(\mathbf{D}^{(1)} \mathbf{G}_{r+1}\right)\right]\right) \\
-\frac{2}{n+1}\left(M \mathbf{G}_{r+1}+n \operatorname{diag}\left(\left[\operatorname{diag}\left(\mathbf{G}_{r+1}\right)\right]^{2}\right)\right) \\
-\frac{\Lambda_{\mu}}{\beta(\beta+1)^{-1}} \operatorname{diag}\left(\left[\operatorname{diag}\left(\mathbf{D}^{(2)} \mathbf{G}_{r+1}\right)\right]\left[\operatorname{diag}\left(\mathbf{T}_{r+1}\right)\right]\right) \\
\mathbf{R e s}_{\boldsymbol{\theta}}=\left\{\begin{array}{c}
-\frac{\Lambda_{\mu}}{\beta(\beta+1)^{-1}} \operatorname{diag}\left(\left[\operatorname{diag}\left(\mathbf{D}^{(1)} \mathbf{G}_{r+1}\right)\right]\left[\operatorname{diag}\left(\mathbf{D}^{(1)} \mathbf{T}_{r+1}\right)\right]\right) \\
+\Lambda_{k} \operatorname{diag}\left(\left[\operatorname{diag}\left(\mathbf{T}_{r+1}\right)\right]\left[\operatorname{diag}\left(\mathbf{D}^{(2)} \mathbf{T}_{r+1}\right)\right]\right)+\Lambda_{k} \operatorname{diag}\left(\left[\operatorname{diag}\left(\mathbf{D}^{(1)} \mathbf{T}_{r+1}\right)\right]^{2}\right)
\end{array}\right\}
\end{array}\right.
$$

For the whole computational domain, we define the following infinite norms:

$$
\begin{aligned}
& \left\|\operatorname{Res}_{\mathbf{g}}\right\|_{\infty}=\operatorname{Max}\left(\left\{\left|\operatorname{Res}_{\mathbf{g}}(i)\right|, \quad \text { where } 1 \leq i \leq N\right\}\right), \\
& \left\|\operatorname{Res}_{\boldsymbol{\theta}}\right\|_{\infty}=\operatorname{Max}\left(\left\{\left|\operatorname{Res}_{\boldsymbol{\theta}}(i)\right|, \quad \text { where } 1 \leq i \leq N\right\}\right) .
\end{aligned}
$$

By applying the above definitions on the constructed vectors $\left(\boldsymbol{R e s}_{\mathbf{g}}, \mathbf{R e s}_{\boldsymbol{\theta}}\right)$ at any advanced iteration step $(r+1)$, the efficiency of the computed results can be authenticated persuasively for every set of the control parameters (i.e., $\eta_{\infty}, N, n, \chi, \beta, \operatorname{Pr}, M, R_{D}, \Lambda_{\mu}$, and $\Lambda_{k}$ ) by utilizing the following convergence criteria:

$$
\left\{\begin{array}{l}
\mathrm{E}_{d}=\operatorname{Max}\left(\left|\left(C_{f r}\right)_{r+1}-\left(C_{f r}\right)_{r}\right|,\left|\left(N u_{r}\right)_{r+1}-\left(N u_{r}\right)_{r}\right|\right) \leq \varepsilon_{d}, \\
\mathbf{E}_{\text {Res }}=\operatorname{Max}\left(\left\|\operatorname{Res}_{\mathbf{g}}\right\|_{\infty},\left\|\operatorname{Res}_{\boldsymbol{\theta}}\right\|_{\infty}\right) \leq \varepsilon_{\text {Res }} .
\end{array}\right.
$$

Based on the above considerations, the iterative numerical schemes converge strongly to higher accurate outcomes, when the systematic errors $E_{d}$ and $E_{\text {Res }}$ are less or equal than the specified convergence tolerance levels $\varepsilon_{d}$ and $\varepsilon_{\text {Res }}$, respectively. On the other hand, the error values $\varepsilon_{d}$ and $\varepsilon_{\text {Res }}$ are generated adequately with magnitude orders of about $10^{-9}$ and $10^{-7}$, correspondingly, to get more precise numerical results. Moreover, the computational cost corresponding to the GDQLLM code execution can be assessed automatically by measuring the CPU time via the MATLAB's Tic-Toc function. In parallel, various proper numerical implementations are carried out via this numerical code to estimate the approximate possible values of $C_{f r}$, $N u_{r},\left\|\operatorname{Res}_{\mathbf{g}}\right\|_{\infty},\left\|\boldsymbol{R e s}_{\boldsymbol{\theta}}\right\|_{\infty}$, and CPU time in Table 1 against various iteration numbers, when $\eta_{\infty}=100, N=250, n=2$, $\chi=0.5, \quad \beta=0.5, \operatorname{Pr}=7, \quad M=2, R_{D}=1, \Lambda_{\mu}=0.8$, and $\Lambda_{k}=1$. From this tabular illustration, it is clear that the proposed numerical method GDQLLM exhibits a faster convergence response, higher flexibility, slighter computational CPU time, and higher robustness, in which the necessary convergence criteria can be attained excellently after just 10 repetitions with a CPU time less than $0.66 \mathrm{~s}$.

\section{Validation of Numerical Results}

More importantly, the engineering applicability of GDQLLM as a new innovative numerical method can be realistically corroborated by making use of the aforementioned accuracy criteria and linking the present flow model with other existing related literature studies. For this purpose, the developed GDQLLM code is scrutinized extensively by comparing appropriately its particular results against those obtained previously by Fang et al. [43], Wang [44], and Mabood et al. [45] for the quantities $-f^{\prime \prime}(0)$ and $-\theta^{\prime}(0)$, when $\beta^{-1}=M=R_{D}=\Lambda_{\mu}=\Lambda_{k}=0$. Sequel to these multiple comparative tests enlisted in Tables 2 and 3 , the accurateness of the GDQLLM results is strengthened more by the SLLM upshots generated by the spectral local linearization method [46]. A quantitative analysis of the tabular data proves that the implemented GDQLLM code exhibits very satisfactory numerical outcomes, which lends a high level of credibility to the present investigation.

\section{Discussion of Results}

After choosing the more appropriate values for the extra parameters $\left(\eta_{\infty}, N\right)$, the GDQLLM results can be obtained successfully, when the number of iterations is achieved a sufficient value $N_{i t}$ providing smaller residual errors with a higher order of precision. For this main purpose, it is preferable to select $\eta_{\infty}=100, N=250$, and $N_{i t}=100$ as the main key parameters controlling the correctness of the present outcomes in all subsequent numerical implementations. By exploiting the accuracy numerical datasets $\left\{\left(f_{N_{i t}}\left(\eta_{i}\right), \theta_{N_{i t}}\left(\eta_{i}\right)\right) / 1 \leq i \leq N\right.$ and $\left.0 \leq \eta_{i} \leq \eta_{\infty}\right\} \quad$ outputted during the completion of the code execution for specified flow parameter values (i.e., $n, \chi, \beta, \operatorname{Pr}, M, R_{D}, \Lambda_{\mu}$, and $\Lambda_{k}$ ), 
TABLE 1: Convergence scrutiny of results in terms of $C_{f r}$ and $N u_{r}$, when $n=2, \chi=0.5, \beta=0.5, \operatorname{Pr}=7, M=2, R_{D}=1, \Lambda_{\mu}=0.8$, and $\Lambda_{k}=1$.

\begin{tabular}{lccccc}
\hline Iterations & $C_{f r}$ & $N u_{r}$ & $\left\|\operatorname{Res}_{g}\right\|_{\infty} \times 10^{7}$ & $\left\|\operatorname{Res}_{\theta}\right\|_{\infty} \times 10^{7}$ & CPU time $(s)$ \\
\hline 1 & 3.702831704 & 2.600241925 & 192683.7059 & 6303740.354 & 0.089999999 \\
2 & 3.535770962 & 2.040731388 & 10688.54111 & 285108.4748 & 0.155999999 \\
3 & 3.530451924 & 2.019105826 & 266.4202916 & 2524.981398 & 0.218999999 \\
4 & 3.530392709 & 2.018913864 & 4.454512438 & 35.36734701 & 0.282999999 \\
5 & 3.530392158 & 2.018911916 & 2.013120419 & 0.429543517 & 0.346999999 \\
10 & 3.530392147 & 2.018911882 & 1.845482356 & 0.002530055 & 0.659999999 \\
15 & 3.530392147 & 2.018911882 & 1.957319967 & 0.003233739 & 0.978999999 \\
20 & 3.530392147 & 2.018911882 & 1.789523721 & 0.001979357 & 1.299999999 \\
25 & 3.530392147 & 2.018911882 & 1.957241065 & 0.002838344 & 1.616999999 \\
30 & 3.530392147 & 2.018911882 & 1.677725372 & 0.002606886 & 1.939999999 \\
35 & 3.530392147 & 2.018911882 & 1.901322070 & 0.003316371 & 2.250000000 \\
40 & 3.530392147 & 2.018911882 & 2.069078297 & 0.003263944 & 2.561999999 \\
45 & 3.530392147 & 2.018911882 & 2.013159302 & 0.002646396 & 2.871999999 \\
50 & 3.530392147 & 2.018911882 & 2.069039035 & 0.002260189 & 3.180999999 \\
\hline
\end{tabular}

TABle 2: Comparison of the present numerical findings for $-f^{\prime \prime}(0)$ with the latest existing literature results, when $\beta^{-1}=M=R_{D}=\Lambda_{k}=\Lambda_{\mu}=0$.

\begin{tabular}{|c|c|c|c|c|c|c|}
\hline \multirow{3}{*}{$n$} & \multirow{2}{*}{\multicolumn{2}{|c|}{$\begin{array}{l}\text { Existing results } \\
\text { Fang et al. }\end{array}$}} & \multicolumn{4}{|c|}{ Present results } \\
\hline & & & \multicolumn{2}{|c|}{ GDQLLM } & \multicolumn{2}{|c|}{ SLLM } \\
\hline & $\chi=0.5$ & $\chi=0.25$ & $\chi=0.5$ & $\chi=0.25$ & $\chi=0.5$ & $\chi=0.25$ \\
\hline 10 & 1.0603 & 1.1433 & 1.060324666 & 1.143320620 & 1.060324666 & 1.143320620 \\
\hline 9 & 1.0589 & 1.1404 & 1.058915793 & 1.140392519 & 1.058915794 & 1.140392519 \\
\hline 7 & 1.0550 & 1.1323 & 1.055044823 & 1.132285178 & 1.055044823 & 1.132285178 \\
\hline 5 & 1.0486 & 1.1186 & 1.048611305 & 1.118590380 & 1.048611306 & 1.118590381 \\
\hline 3 & 1.0359 & 1.0905 & 1.035868281 & 1.090492254 & 1.035868282 & 1.090492254 \\
\hline 2 & 1.0234 & - & 1.023407744 & 1.061402504 & 1.023407744 & 1.061402505 \\
\hline 1 & 1.0000 & 1.0000 & 1.000000000 & 1.000000000 & 1.000000000 & 1.000000000 \\
\hline $1 / 2$ & 0.9799 & 0.9338 & 0.979944970 & 0.933825409 & 0.979944970 & 0.933825410 \\
\hline 0 & 0.9576 & 0.7843 & 0.957643151 & 0.784279230 & 0.957643151 & 0.784279231 \\
\hline$-1 / 3$ & 1.0000 & 0.5000 & 1.000000000 & 0.500000000 & 1.000000000 & 0.500000000 \\
\hline
\end{tabular}

TABLE 3: Comparison of the present numerical findings for $-\theta^{\prime}(0)$ with the latest existing literature works, when $n=1$ and $\beta^{-1}=M=R_{D}=\Lambda_{k}=\Lambda_{\mu}=0$.

\begin{tabular}{lcccr}
\hline Pr & \multicolumn{3}{c}{ Existing results } & \multicolumn{2}{c}{ Present results } \\
& Wang [44] & Mabood et al. [45] & GDQLLM & 0.065622569 \\
0.07 & 0.0656 & 0.0665 & 0.169088573 & 0.065622569 \\
0.2 & 0.1691 & 0.1691 & 0.453916157 & 0.453916157 \\
0.7 & 0.4539 & 0.4539 & 0.911357683 & 0.911357683 \\
2 & 0.9114 & 0.9114 & 1.895403258 & 1.895403258 \\
7 & 1.8954 & 1.8954 & 3.353904143 & 3.353904143 \\
20 & 3.3539 & 3.3539 & 6.462199430 & \\
70 & 6.4622 & 6.4622 & 6.462199531 \\
\hline
\end{tabular}

the fluid flow properties and heat transfer characteristics are revealed throughout the medium in terms of velocity and temperature fields, as well as locally via the skin friction coefficient and Nusselt number at the slender sheet. These physical features are presented and discussed via numerous graphical and tabular displays by taking $n=2, \chi=0.5$, $\beta=0.5, \operatorname{Pr}=7, M=2, R_{D}=1, \Lambda_{\mu}=0.8$, and $\Lambda_{k}=1$ as default flow parameter values, unless otherwise indicated. Furthermore, to elucidate exhaustive physical insights from the graphical representations, the velocity and temperature profiles are clipped from 0 to 8 to show the impacts of every embedded parameter more visibly and avoid the overlap between the curves in each graphical illustration.

As exhibited below, the graphical and tabular representations displayed in Figures 2-10 and Tables 4 and 5 reflect completely the fluid motion and heat transfer characteristics (i.e., velocity profiles, temperature profiles, skin friction coefficients, heat transfer rates, velocity boundary 


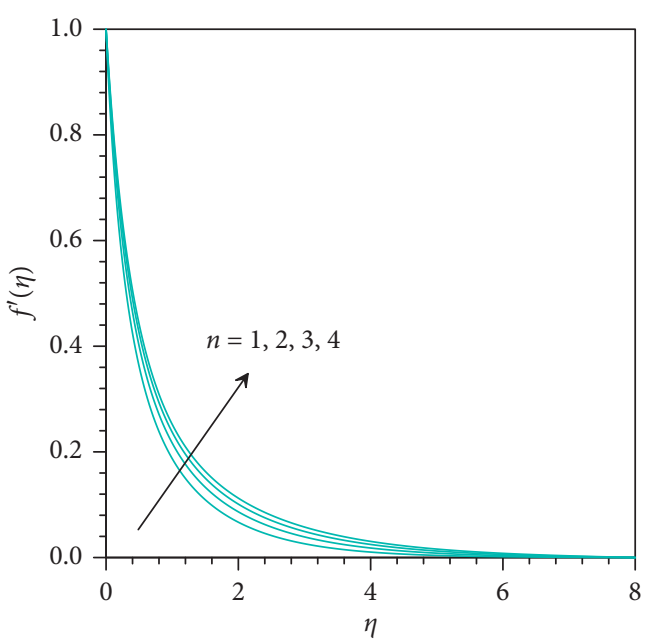

(a)

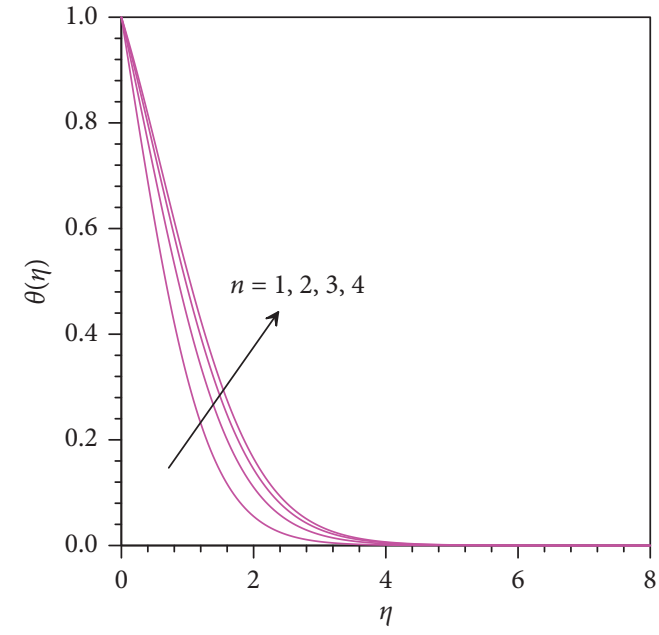

(b)

Figure 2: Influence of $n$ on $f^{\prime}(\eta)$ and $\theta(\eta)$.

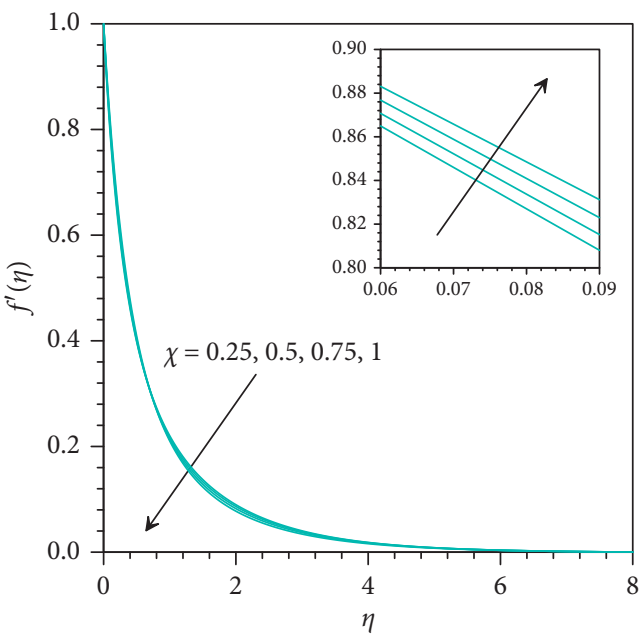

(a)

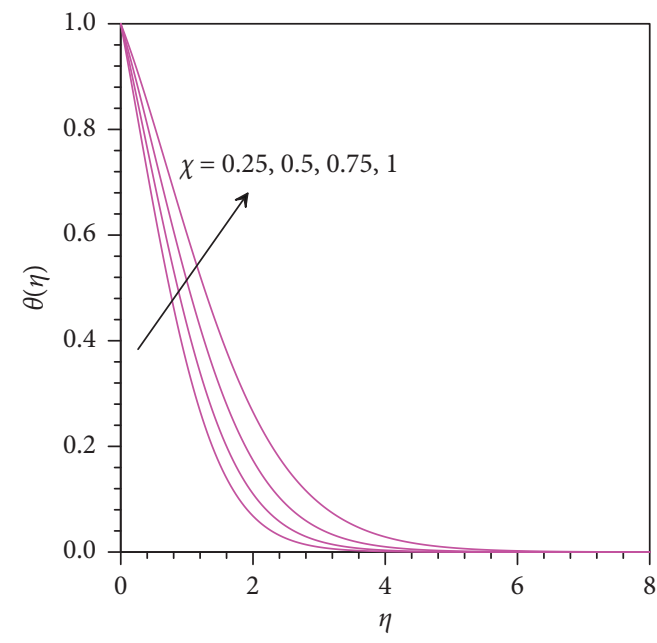

(b)

Figure 3: Influence of $\chi$ on $f^{\prime}(\eta)$ and $\theta(\eta)$.

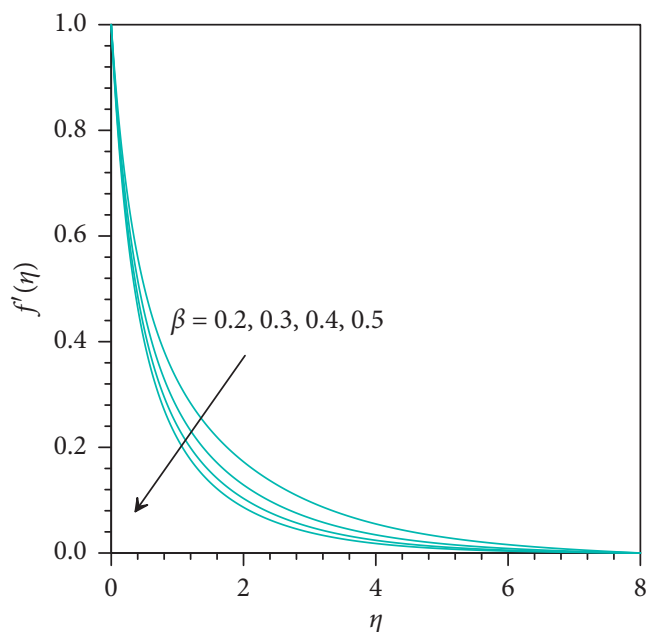

(a)

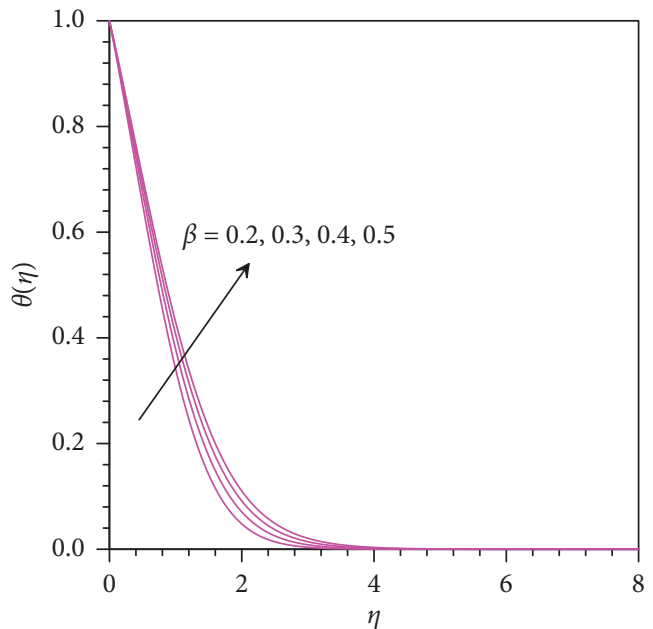

(b)

Figure 4: Influence of $\beta$ on $f^{\prime}(\eta)$ and $\theta(\eta)$. 


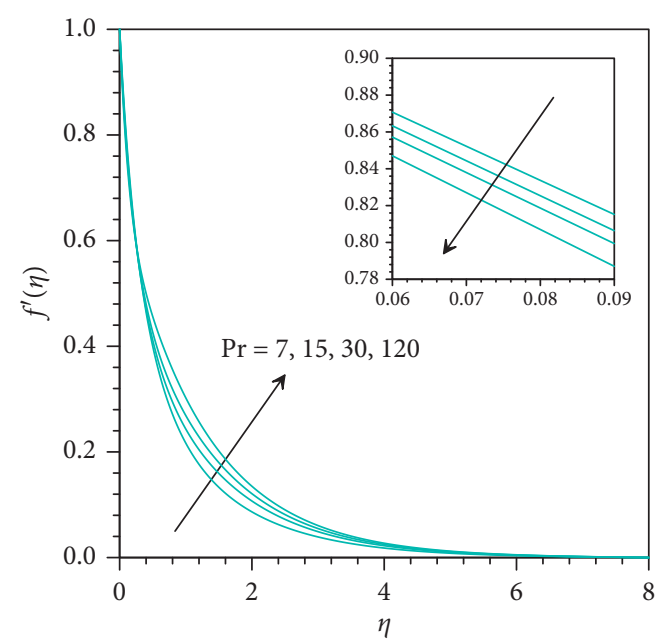

(a)

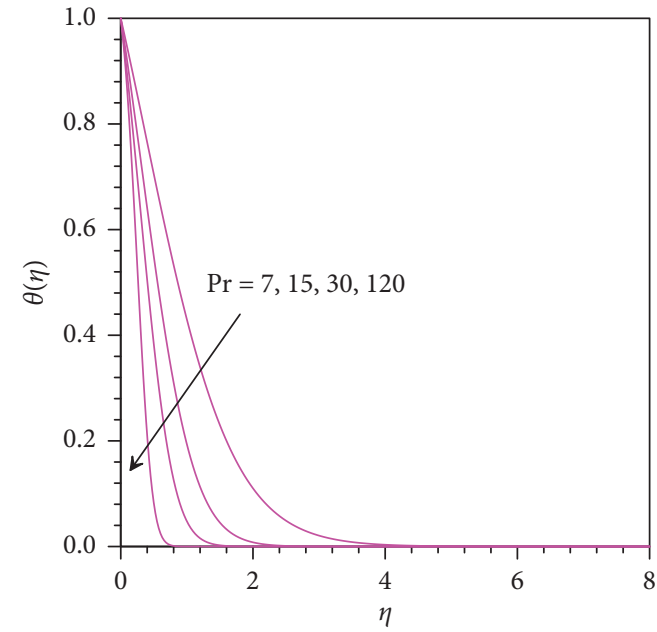

(b)

Figure 5: Influence of $\operatorname{Pr}$ on $f^{\prime}(\eta)$ and $\theta(\eta)$.

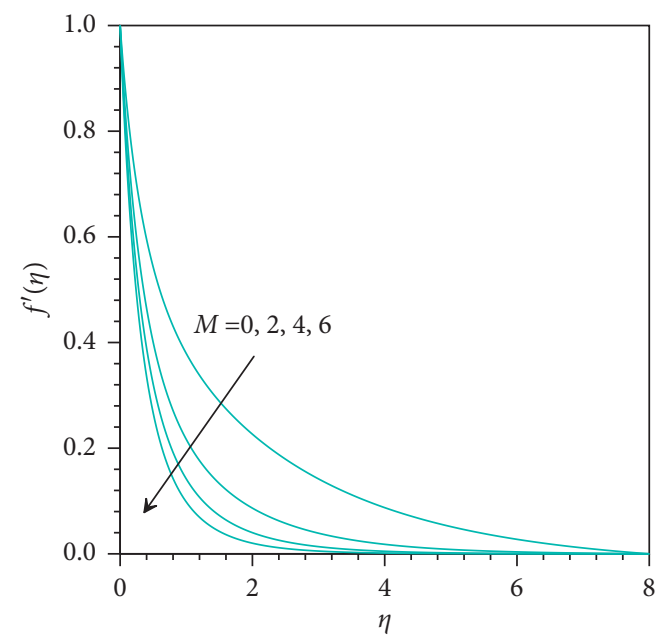

(a)

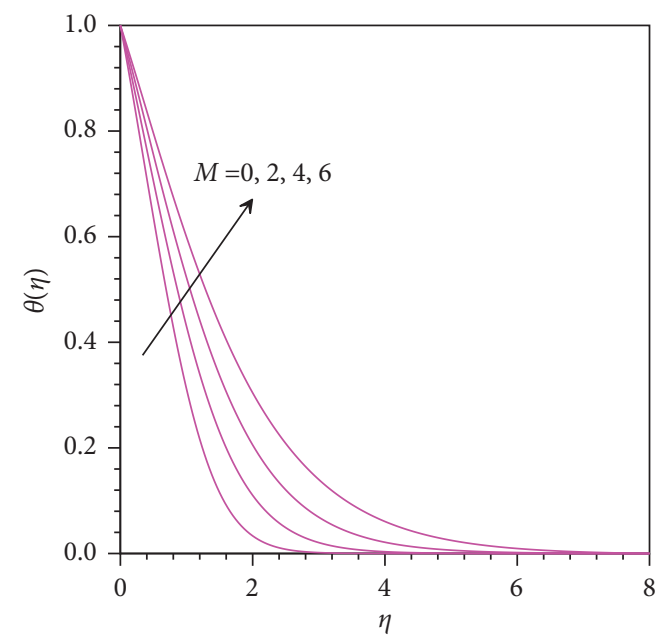

(b)

Figure 6: Influence of $M$ on $f^{\prime}(\eta)$ and $\theta(\eta)$.
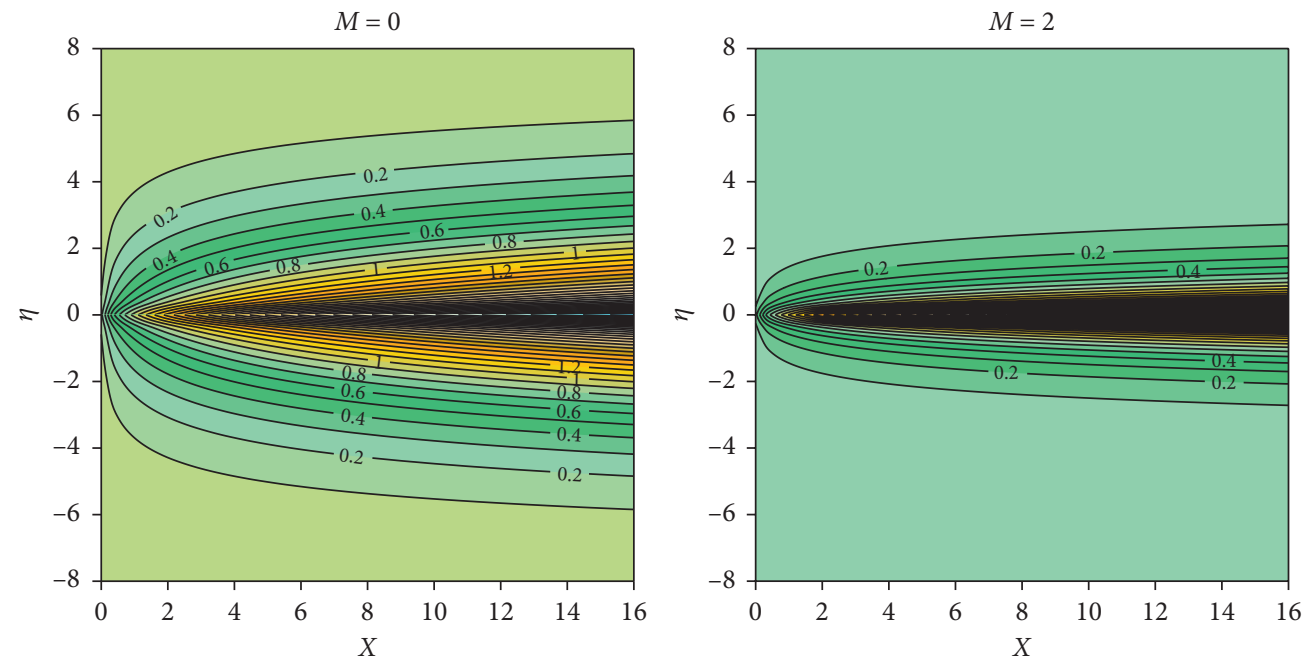

(a)

Figure 7: Continued. 

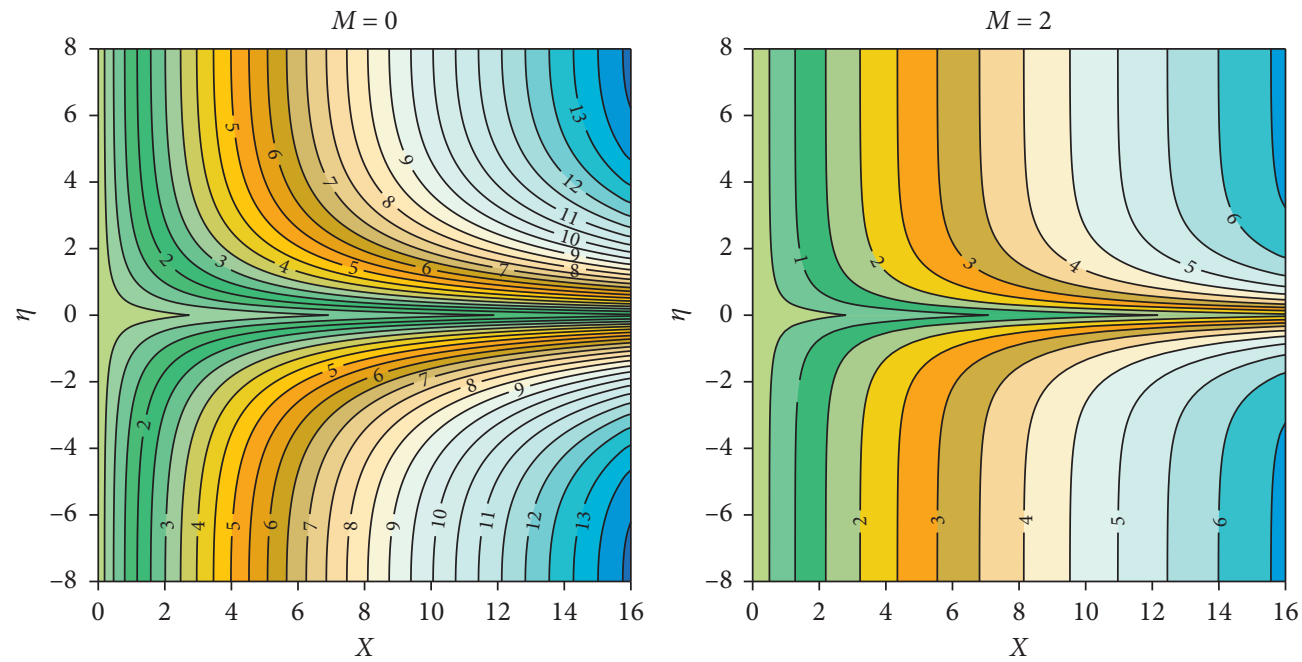

(b)

Figure 7: Effect of $M$ on (a) velocity boundary layers and (b) streamlines.

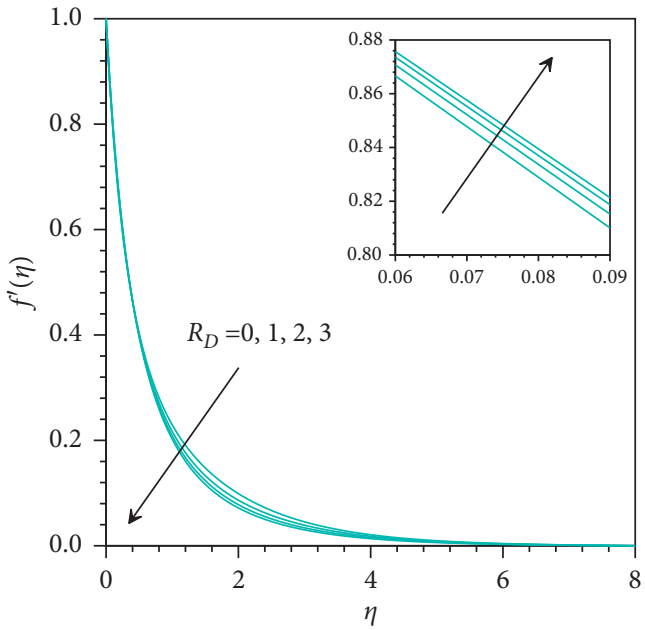

(a)

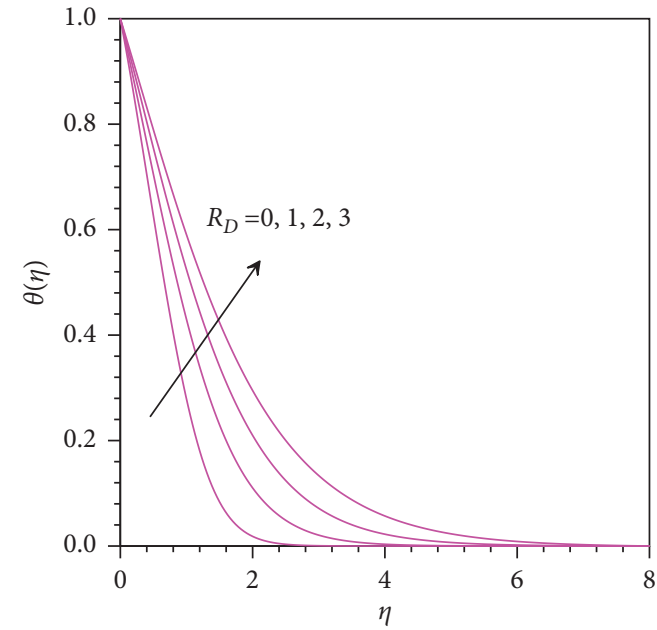

(b)

Figure 8: Influence of $R_{D}$ on $f^{\prime}(\eta)$ and $\theta(\eta)$.

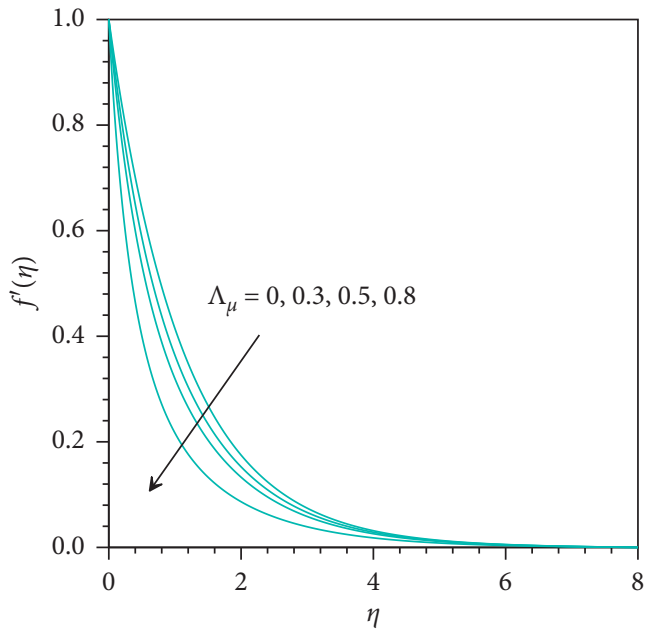

(a)

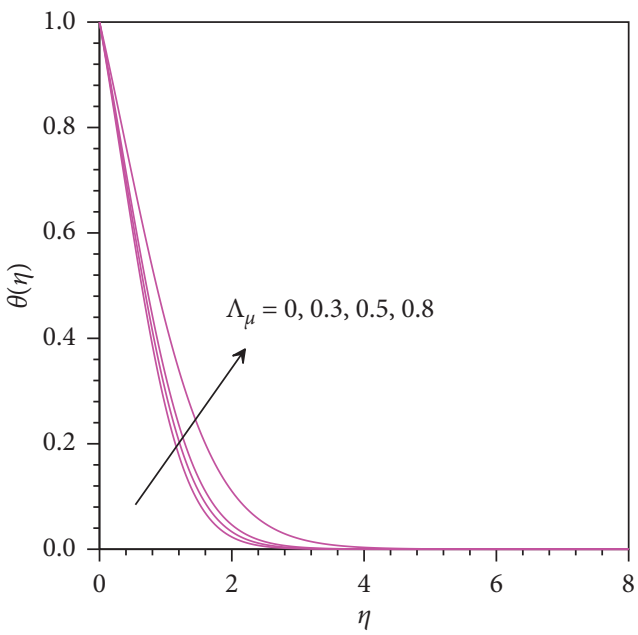

(b)

Figure 9: Influence of $\Lambda_{\mu}$ on $f^{\prime}(\eta)$ and $\theta(\eta)$. 


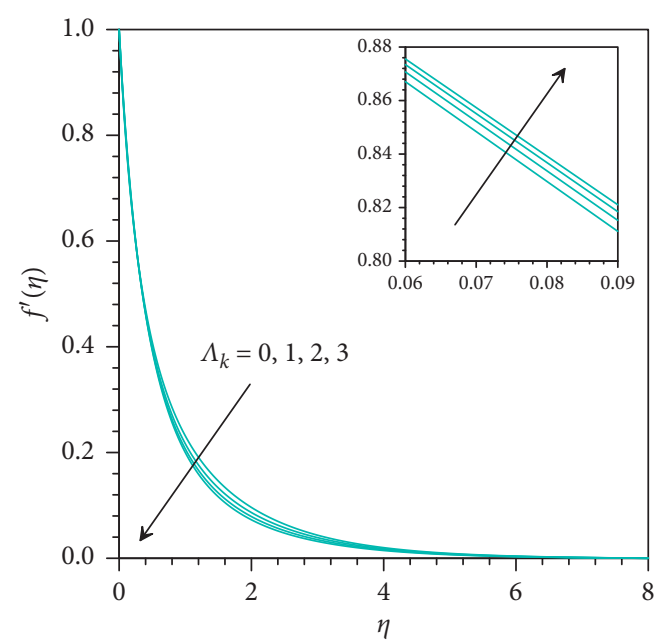

(a)

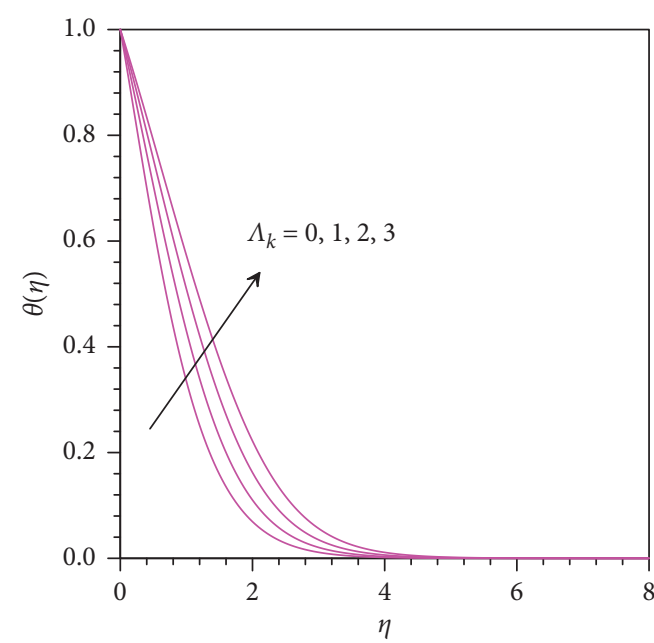

(b)

Figure 10: Influence of $\Lambda_{k}$ on $f^{\prime}(\eta)$ and $\theta(\eta)$.

layers, and streamlines) of the studied non-Newtonian fluid against sundry influencing parameters, namely, velocity power parameter $n$, wall thickness parameter $\chi$, Casson fluid parameter $\beta$, Prandtl number $\operatorname{Pr}$, magnetic parameter $M$, radiation parameter $R_{D}$, variable viscosity parameter $\Lambda_{\mu}$, and variable thermal conductivity parameter $\Lambda_{k}$. Generally, it is observed that the dimensionless profiles of the horizontal velocity distributions $\left\{f^{\prime}\left(\eta_{i}\right)=g\left(\eta_{i}\right) / 1 \leq i \leq N\right.$ and $\left.0 \leq \eta_{i} \leq \eta_{\infty}\right\}$ are monotonically declining functions and tends asymptotically to the vanishing boundary condition $f^{\prime}\left(\eta \longrightarrow \eta_{\infty}\right)=0$ as long as the similarity variable $\eta$ exceeds a certain distance $\eta=\delta(\leq 8)$, which indicates the approximate value of the velocity boundary layer thickness.

The probable evolution of the dimensionless velocity and temperature profiles $\left(f^{\prime}(\eta), \theta(\eta)\right)$ in response to the increasing change in the values of the power index parameter $n$ are delineated quite clearly in Figure 2. Graphically, it is shown that the dynamical parameter $n$ exhibits a heightening effect towards the thermohydrodynamic profiles $\left(f^{\prime}(\eta), \theta(\eta)\right)$ with a significant widening in the thermohydrodynamic boundary layer thicknesses. These circumstances can be explained more by the higher increment in the magnitude of the nonlinear stretching velocity of the heated slender sheet as long as the velocity exponent $n$ is upsurged, which leads to thicker thermohydrodynamic boundary layers.

Additionally, Figure 3 is plotted to elucidate the sensitive effect of the wall thickness parameter $\chi$ on the dimensionless velocity and temperature profiles $\left(f^{\prime}(\eta), \theta(\eta)\right)$. Seemingly, the fluid flow shows a dual behaviour concerning the geometrical parameter $\chi$ depending on the nearness or the farness from the stretching sheet, whereas a thermal enhancement is disclosed throughout the fluidic medium. After analysing carefully the graphical results highlighted in Figure 3, it is found that the higher values of the wall thickness parameter $\chi$ cause a tenuous increase in the fluid velocity near the stretching sheet; however, a slower fluid motion is observed far from the horizontal boundary to get almost a thinner velocity boundary layer. Since the velocity exponent $n$ is greater than the unit value in this problem (i.e., $n=2$ ), the function $f(\eta)$ takes negative values near the stretching sheet and decreases with the increasing values of the wall thickness parameter $\chi$, due to the imposed boundary condition $f(0)=((1-n) \chi /(1+n))$. So, the rising trend revealed for the velocity profile $f^{\prime}(\eta)$ near the stretching sheet against the increasing values of the geometrical parameter $\chi$ can be explicated quite clearly by invoking the vanishing boundary condition for the dimensional transversal velocity $v(x, y)$, considering the positive sign of the dimensionless horizontal velocity $f^{\prime}(\eta)$ and making use of the transformations defined in equations (7) and (13) for the expression of $v(x, y)$. Furthermore, the thermal improvement noticed in the fluidic medium can be clarified by the blowing impact arising from the augmentation in the wall thickness parameter $\chi$.

Rheologically, the Casson parameter $\beta$ reflects the viscoplastic non-Newtonian behaviour of the present boundary layer flow. Moreover, the Casson viscoplastic fluid model can be reduced to the Newtonian viscous fluid case just by escalating the rheological parameter $\beta$ to an extremely higher value (i.e., $\beta \longrightarrow \infty$ ). Also, an increase in the Casson parameter $\beta$ rises the plastic dynamic viscosity of the Casson fluid and decreases the resulting yield stress. Thereby, a viscous resistance is induced to the flow in the fluidic medium. For this reason, the fluid velocity lessens with the escalating values of the Casson viscoplastic parameter $\beta$, while an opposite effect is detected for the fluid temperature as portrayed in Figure 4. Consequently, the Casson parameter $\beta$ has the proclivity to suppress the velocity boundary layer region and heighten the thermal boundary layer thickness. 
TABLE 4: Various numerical results for $C_{f r}$ via GDQLLM and SLLM.

\begin{tabular}{|c|c|c|c|c|c|c|c|c|c|c|c|}
\hline \multirow{2}{*}{$n$} & \multirow[b]{2}{*}{$\chi$} & \multirow{2}{*}{$\beta$} & \multirow{2}{*}{$\operatorname{Pr}$} & \multirow{2}{*}{$M$} & \multirow{2}{*}{$R_{D}$} & \multirow{2}{*}{$\Lambda_{\mu}$} & \multirow{2}{*}{$\Lambda_{k}$} & \multicolumn{2}{|c|}{$C_{f r}$} & \multicolumn{2}{|c|}{ Slope (linear regression) } \\
\hline & & & & & & & & GDQLLM & SLLM & GDQLLM & SLLM \\
\hline 1 & \multirow{4}{*}{0.5} & \multirow{4}{*}{0.5} & \multirow{4}{*}{7} & \multirow{4}{*}{2} & \multirow{4}{*}{1} & \multirow{4}{*}{0.8} & \multirow{4}{*}{1} & 3.438280856 & 3.438280856 & \multirow{4}{*}{0.15161435} & \multirow{4}{*}{0.15161436} \\
\hline 2 & & & & & & & & 3.530392147 & 3.530392147 & & \\
\hline 3 & & & & & & & & 3.697942773 & 3.697942774 & & \\
\hline \multirow[t]{2}{*}{4} & & & & & & & & 3.887811830 & 3.887811831 & & \\
\hline & 0.25 & \multirow{4}{*}{0.5} & \multirow{4}{*}{7} & \multirow{4}{*}{2} & \multirow{4}{*}{1} & \multirow{4}{*}{0.8} & & 3.739895693 & 3.739895694 & & \\
\hline \multirow{4}{*}{2} & 0.5 & & & & & & 1 & 3.530392147 & 3.530392147 & -083621408 & -083621408 \\
\hline & 0.75 & & & & & & 1 & 3.320809783 & 3.320809783 & -0.85021408 & -0.83021400 \\
\hline & 1 & & & & & & & 3.112911418 & 3.112911419 & & \\
\hline & & 0.2 & & & & & & 5.586732509 & 5.586732511 & & \\
\hline 2 & 05 & 0.3 & 7 & 2 & 1 & 08 & 1 & 4.505453930 & 4.505453930 & -676385072 & -676385072 \\
\hline 2 & 0.5 & 0.4 & 7 & 2 & 1 & 0.8 & 1 & 3.910624300 & 3.910624301 & $-0.163850 / 2$ & $-0 . / 63850 / 2$ \\
\hline & & 0.5 & & & & & & 3.530392147 & 3.530392147 & & \\
\hline & & & 7 & & & & & 3.530392147 & 3.530392147 & & \\
\hline & 0.5 & & 15 & & & & 1 & 3.818808341 & 3.818808341 & 000638303 & 000638393 \\
\hline 2 & 0.5 & 0.5 & 30 & 2 & 1 & 0.8 & 1 & 4.071512266 & 4.071512266 & 0.00638393 & 0.00638393 \\
\hline & & & 120 & & & & & 4.404651535 & 4.404651535 & & \\
\hline & & & & 0 & & & & 2.583396991 & 2.583396991 & & \\
\hline 2 & 0.5 & 0.5 & 7 & 2 & 1 & 08 & 1 & 3.530392147 & 3.530392147 & 034450398 & 034450298 \\
\hline 2 & 0.3 & 0.5 & 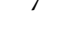 & 4 & 1 & 0.8 & 1 & 4.161445943 & 4.161445944 & 0.34450290 & 0.04450290 \\
\hline & & & & 6 & & & & 4.669732284 & 4.669732284 & & \\
\hline & & & & & 0 & & & 3.678361324 & 3.678361324 & & \\
\hline 2 & 0.5 & 0.5 & 7 & 2 & 1 & 08 & 1 & 3.530392147 & 3.530392147 & -010859003 & -010859003 \\
\hline 2 & 0.5 & 0.5 & 7 & 2 & 2 & 0.8 & 1 & 3.427353550 & 3.427353550 & -0.10859003 & -0.10859003 \\
\hline & & & & & 3 & & & 3.350740762 & 3.350740762 & & \\
\hline & & & & & & 0 & & 6.578902921 & 6.578902924 & & \\
\hline 2 & 05 & 0.5 & 7 & 2 & 1 & 0.3 & 1 & 5.801347590 & 5.801347593 & -378728476 & -378728476 \\
\hline 2 & 0.5 & 0.5 & 1 & 2 & 1 & 0.5 & 1 & 5.118622501 & 5.118622503 & $-3 . / 8 / 284 / 0$ & $-3 . / 8 / 284 / 6$ \\
\hline & & & & & & 0.8 & & 3.530392147 & 3.530392147 & & \\
\hline & & & & & & & 0 & 3.687725276 & 3.687725277 & & \\
\hline 2 & 05 & 0.5 & 7 & 2 & 1 & 08 & 1 & 3.530392147 & 3.530392147 & & \\
\hline 2 & 0.5 & 0.5 & 7 & 2 & 1 & 0.8 & 2 & 3.425271152 & 3.425271152 & $-0.11216 / 01$ & -0.11216701 \\
\hline & & & & & & & 3 & 3.348875572 & 3.348875573 & & \\
\hline
\end{tabular}

Via a physical standpoint, the temperature dependency involved in the dynamic viscosity and thermal conductivity expressions causes a fully mathematical coupling between the conservation equations (i.e., momentum and energy equations) and engenders an implicit effect of the Prandtl number Pr on the fluid flow and heat transfer features as depicted in Figure 5. Due to the augmenting values of the Prandtl number Pr, the temperature of the medium diminishes and the fluid slows down slightly near the isothermal horizontal boundary, while an accelerating impact is achieved after a certain distance from the sheet, which in turn provides an enhancement in the velocity boundary layer thickness. Besides, the Prandtl number Pr illustrates a direct link between the fluid motion inside the velocity boundary layer region and its corresponding temperature, since it represents the ratio between the kinematic viscosity $v(=\mu / \rho)$ of the viscoplastic medium to its thermal diffusivity $\alpha\left(=k /\left(\rho C_{P}\right)\right)$. Therefore, an enhancement in the Prandtl number Pr weakens the thermal diffusive influence in front of the momentum diffusive impact. As a result of the viscous dominance, the thermal boundary layer becomes more thinner and the fluid motion shows a weak deceleration near the stretching sheet, whilst an opposite behaviour is perceived whenever we get away from the isothermal sheet because of the thermophysical interference noticed between the impacts of the variable properties arising from the temperature-dependent parameters $\left(\Lambda_{\mu}, \Lambda_{k}\right)$ introduced in the governing equations.

On the one hand, the illustrative variations in the dimensionless velocity and temperature profiles $\left(f^{\prime}(\eta), \theta(\eta)\right)$ against the varying values of the magnetic parameter $M$ are established graphically in Figure 6. From this graphical visualization, it is evident that an increase in this flow parameter leads to a lessening in the fluid velocity. This slowing behaviour demonstrated for the fluid velocity towards the intensifying values of the magnetic parameter $M$ can be attributed to the existence of Lorentz forces, which are happened in the electrically conducting fluidic medium due to the presence of an external magnetic field applied transversely to the fluid flow. Besides, these magnetic forces act meaningfully as a resistive factor by weakening the velocity boundary layer thickness as evidenced in Figure 7(a) and enlarging its corresponding thermal width. These facts are occurred because of both the deceleration in the fluid motion and the elevation in its temperature. On the other hand, Figure 7 (b) proves that the flow patterns are affected 
TABLe 5: Various numerical results for $N u_{r}$ via GDQLLM and SLLM.

\begin{tabular}{|c|c|c|c|c|c|c|c|c|c|c|c|}
\hline \multirow{2}{*}{$n$} & \multirow[b]{2}{*}{$\chi$} & \multirow{2}{*}{$\beta$} & \multirow{2}{*}{$\operatorname{Pr}$} & \multirow{2}{*}{$M$} & \multirow{2}{*}{$R_{D}$} & \multirow{2}{*}{$\Lambda_{\mu}$} & \multirow{2}{*}{$\Lambda_{k}$} & \multicolumn{2}{|c|}{$N u_{r}$} & \multicolumn{2}{|c|}{ Slope (linear regression) } \\
\hline & & & & & & & & GDQLLM & SLLM & GDQLLM & SLLM \\
\hline 1 & \multirow{4}{*}{0.5} & \multirow{4}{*}{0.5} & \multirow{4}{*}{7} & \multirow{4}{*}{2} & \multirow{4}{*}{1} & \multirow{4}{*}{0.8} & \multirow{4}{*}{1} & 2.328014017 & 2.328014017 & \multirow{4}{*}{-0.12682904} & \multirow{4}{*}{-0.12682904} \\
\hline 2 & & & & & & & & 2.018911882 & 2.018911882 & & \\
\hline 3 & & & & & & & & 1.935477626 & 1.935477626 & & \\
\hline \multirow[t]{2}{*}{4} & & & & & & & & 1.933061942 & 1.933061942 & & \\
\hline & 0.25 & \multirow{4}{*}{0.5} & \multirow{4}{*}{7} & \multirow{4}{*}{2} & \multirow{4}{*}{1} & \multirow{4}{*}{0.8} & & 2.480970502 & 2.480970502 & & \\
\hline \multirow{4}{*}{2} & 0.5 & & & & & & & 2.018911882 & 2.018911882 & & \\
\hline & 0.75 & & & & & & 1 & 1.588471020 & 1.588471020 & -1.7176862 & -1.7176862 \\
\hline & 1 & & & & & & & 1.193476939 & 1.193476939 & & \\
\hline & & 0.2 & & & & & & 2.359394987 & 2.359394987 & & \\
\hline & & 0.3 & & & & & & 2.212663880 & 2.212663880 & & \\
\hline 2 & 0.5 & 0.4 & 7 & 2 & 1 & 0.8 & 1 & 2.104105813 & 2.104105813 & -1.13000738 & -1.13000738 \\
\hline & & 0.5 & & & & & & 2.018911882 & 2.018911882 & & \\
\hline & & & 7 & & & & & 2.018911882 & 2.018911882 & & \\
\hline & & & 15 & & & & & 2.926415233 & 2.926415233 & & \\
\hline 2 & 0.5 & 0.5 & 30 & 2 & 1 & 0.8 & 1 & 3.704016463 & 3.704016463 & 0.01118628 & 0.01118628 \\
\hline & & & 120 & & & & & 3.821481472 & 3.821481472 & & \\
\hline & & & & 0 & & & & 2.478226379 & 2.478226379 & & \\
\hline & & & & 2 & & & & 2.018911882 & 2.018911882 & & \\
\hline 2 & 0.5 & 0.5 & 7 & 4 & 1 & 0.8 & 1 & 1.682903617 & 1.682903617 & -0.17754976 & $-0.177549 / 6$ \\
\hline & & & & 6 & & & & 1.406564006 & 1.406564006 & & \\
\hline & & & & & 0 & & & 1.609470214 & 1.609470214 & & \\
\hline & & & & & 1 & & & 2.018911882 & 2.018911882 & & \\
\hline 2 & 0.5 & 0.5 & 7 & 2 & 2 & 0.8 & 1 & 2.293768176 & 2.293768176 & 0.29129307 & 0.29129307 \\
\hline & & & & & 3 & & & 2.488828352 & 2.488828352 & & \\
\hline & & & & & & 0 & & 2.688132140 & 2.688132140 & & \\
\hline 2 & 0.5 & 0.5 & 7 & 2 & 1 & 0.3 & 1 & 2.561008484 & 2.561008484 & -0.82609556 & -0.82609556 \\
\hline & & & & & & 0.5 & 1 & 2.429164586 & 2.429164586 & & \\
\hline & & & & & & 0.8 & & 2.018911882 & 2.018911882 & & \\
\hline & & & & & & & 0 & 1.759358147 & 1.759358147 & & \\
\hline 2 & 0.5 & 0.5 & 7 & 2 & 1 & 0.8 & 1 & 2.018911882 & 2.018911882 & 0.20547491 & 0.20547491 \\
\hline & & & & & & & 2 & 2.218779372 & 2.218779372 & & \\
\hline & & & & & & & 3 & 2.377652036 & 2.377652036 & & \\
\hline
\end{tabular}

noticeably by the presence of an externally applied magnetic, in which the values of the dimensionless stream function decline notably, due to the slowing down of the fluid velocity within the momentum boundary layer. Moreover, the streamline shapes become wider and tend to be parallel as long as we go away from the stretching sheet.

Energetically, the thermal radiation parameter $R_{D}\left(=\left(16 \sigma_{e} T_{\infty}^{3}\right) /\left(3 \beta_{R} k\right)\right)$ represents the effective contribution rate between the radiative and conductive heat transports. So, an intensification in the radiation parameter $R_{D}$ causes an increase in the radiative manner of heat transfer, which in turn leads to a strengthening effect on the fluid motion near the hot slender sheet with an enhancement in both the temperature field and its boundary layer thickness as emphasized graphically in Figure 8. Also, it is witnessed that the fluid motion shows a slowing behaviour towards an upsurge in the values of the radiation parameter $R_{D}$ when going away from the stretching sheet, which implies a reduction in the velocity boundary layer thickness.

Additionally, the inevitable features of the variable viscosity parameter $\Lambda_{\mu}(>0)$ on the dimensionless velocity and temperature distributions $\left\{\left(f^{\prime}\left(\eta_{i}\right), \theta\left(\eta_{i}\right)\right) / 1 \leq i \leq N\right.$ and $\left.0 \leq \eta_{i} \leq \eta_{\infty}\right\}$ are underlined obviously in Figure 9. From this graphical representation, it is revealed that the rising values of the variable viscosity parameter $\Lambda_{\mu}$ have a decelerating impact on the horizontal fluid motion, while a thermal enhancement is depicted in the medium due to this increasing variation in the control parameter $\Lambda_{\mu}$. As mentioned before, the linear temperature dependency adopted for the physical propriety $\mu_{T}$ implies that the variable viscosity parameter $\Lambda_{\mu}$ has a declining effect on the dynamic viscosity $\mu_{T}$, which results in turn to a shearthinning rheological characteristic for the studied nonNewtonian fluid. Indeed, the increasing values of the variable viscosity parameter $\Lambda_{\mu}$ cause a stronger temperature difference $\left(T_{w}-T_{\infty}\right)$ between the stretching sheet and the free-stream fluid. So, larger values of the variable viscosity parameter $\Lambda_{\mu}$ increase the fluidity of the medium and strengthen the fluid motion in the vertically upward direction as compared with its horizontal flow. For this reason, a diminishing trend is seen for the hydrodynamic boundary layer thickness, while an opposite upshot is found for the thermal boundary layer thickness.

Unlike the aforediscussed results for the variable viscosity parameter $\Lambda_{\mu}$, the consequences of supposing a linear temperature dependency for the thermal 
conductivity $k_{T}$ on the dimensionless velocity and temperature profiles $\left(f^{\prime}(\eta), \theta(\eta)\right)$ are underscored in Figure 10. Generally, an elevation in the variable thermal conductivity parameter $\Lambda_{k}$ leads to an important enrichment in the fluid thermal conductivity, which in turn improves the fluid temperature. However, a significant depletion in the dynamic viscosity $\mu_{T}$ is inspected within the fluidic medium due to the augmentation in its liquidity. Keeping in mind that the flow and heat transport mechanisms are notably influenced by the thermal conductivity along the transverse direction, the fluid motion is reinforced near the heated starching sheet with the increasing values of the variable thermal conductivity parameter $\Lambda_{k}$, while a reverse trend is seen away from the stretching sheet because of the vertical motion tendency. Consequently, the variable thermal conductivity parameter $\Lambda_{k}$ has a diminishing influence on the hydrodynamic boundary layer thickness, whilst an enlargement is noticed for the thermal boundary layer zone.

By applying the statistical procedure SLRM [47-49] on the accurate GDQLLM and SLLM datasets generated for the present viscoplastic flow model, the fluid flow and heat transfer features against the rising values of each involved flow parameter (i.e., $n, \chi, \beta, \operatorname{Pr}, M, R_{D}, \Lambda_{\mu}$, and $\Lambda_{k}$ ) are studied systematically based on the reduced physical quantities $C_{f r}$ and $N u_{r}$ arranged in Tables 4 and 5. From these tabular demonstrations, it is found that the surface frictional effect arising from the amplifying values of the engineering quantity $C_{f r}$ can be minimized either by intensifying the values of wall thickness parameter $\chi$, Casson fluid parameter $\beta$, radiation parameter $R_{D}$, variable viscosity parameter $\Lambda_{\mu}$, and variable thermal conductivity parameter $\Lambda_{k}$ or by lowering the values of velocity power parameter $n$, Prandtl number Pr, and magnetic parameter $M$. However, an enhancement in the surface heat transfer rate can be attained either by mounting the values of the parameters $\operatorname{Pr}, R_{D}$, and $\Lambda_{k}$ or by lessening the values of the parameters $n, \chi, \beta, M$, and $\Lambda_{\mu}$.

\section{Concluding Remarks}

Numerous interesting physical and mathematical insights have been realistically drawn for the present MHD radiativeconvective flow problem in the case of an enhanced Casson viscoplastic fluid having temperature-dependent thermophysical properties (i.e., dynamic viscosity and thermal conductivity) and moving horizontally over an irregular isothermal stretching sheet by employing an innovative numerical approach, called the generalized differential quadrature local linearization method (GDQLLM). In this framework, noteworthy GDQLLM outcomes have been derived from this numerical investigation, which can be itemized as follows:

(i) The proposed GDQLLM algorithm exhibits excellent robustness and flexibility towards the present nonlinear flow problem

(ii) The GDQLLM results can be fastly reached with a higher level of precision with a scarce number of iterations (iii) The fluid velocity profiles show a decreasing behaviour against the flow parameters $\beta, M$, and $\Lambda_{\mu}$, while a hastening trend is noticed for larger values of the parameter $n$

(iv) The fluid motion can be strengthened slightly near the stretching sheet either by escalating the values of $\chi, R_{D}$, and $\Lambda_{k}$ or by lessening only the value of $\operatorname{Pr}$, whilst opposite dynamical features are revealed for these parameters away from the stretching sheet

(v) Larger values of $n, \chi, \beta, M, R_{D}, \Lambda_{\mu}$, and $\Lambda_{k}$ yield a significant enhancement in the fluid temperature, while a reverse thermal feature is depicted for the mounting values of $\mathrm{Pr}$

(vi) The strength of the resulting surface drag forces can be reduced either by escalating the values of $\chi, \beta$, $R_{D}, \Lambda_{\mu}$, and $\Lambda_{k}$ or by weakening the values of $n, \operatorname{Pr}$, and $M$

(vii) An enrichment in the surface heat transfer rate can be achieved either by intensifying the values of $\mathrm{Pr}$, $R_{D}$, and $\Lambda_{k}$ or by lessening the values of $n, \chi, \beta, M$, and $\Lambda_{\mu}$

\section{Data Availability}

The data of the present problem cannot be shared due to confidentiality requirements.

\section{Conflicts of Interest}

The authors declare that they have no conflicts of interest.

\section{References}

[1] S. Bilal, M. Sohail, R. Naz, M. Y. Malik, and M. Alghamdi, "Upshot of ohmically dissipated Darcy-Forchheimer slip flow of magnetohydrodynamic Sutterby fluid over radiating linearly stretched surface in view of Cash and Carp method," Applied Mathematics and Mechanics, vol. 40, no. 6, pp. 861876, 2019.

[2] M. Bibi, A. Zeeshan, M. Y. Malik, and K. U. Rehman, "Numerical investigation of the unsteady solid-particle flow of a tangent hyperbolic fluid with variable thermal conductivity and convective boundary," The European Physical Journal Plus, vol. 134, no. 6, pp. 1-14, 2019.

[3] R. Mohebbi, A. A. Delouei, A. Jamali, M. Izadi, and A. A. Mohamad, "Pore-scale simulation of non-Newtonian power-law fluid flow and forced convection in partially porous media: thermal lattice Boltzmann method," Physica A: Statistical Mechanics and Its Applications, vol. 525, pp. 642656, 2019.

[4] Y. Liu, C. Shi, Q. Yuan et al., "An amendment of rotation speed-torque transformation equation for the HerschelBulkley model in wide-gap coaxial cylinders rheometer," Construction and Building Materials, vol. 237, Article ID 117530, 2020.

[5] W. H. Herschel and R. Bulkley, "Konsistenzmessungen von gummi-benzollösungen," Kolloid-Zeitschrift, vol. 39, no. 4, pp. 291-300, 1926.

[6] C. Zhu, Z. Yu, X. Shao, and J. Deng, "Interface-resolved numerical simulations of particle-laden turbulent flows in a 
vertical channel filled with Bingham fluids," Journal of Fluid Mechanics, vol. 883, pp. 1-29, 2020.

[7] E. C. Bingham, Fluidity and Plasticity, McCraw-Hill B. Co., Inc., New York, NY, USA, 1922.

[8] H. Waqas, S. U. Khan, M. M. Bhatti, and M. Imran, "Significance of bioconvection in chemical reactive flow of magnetized Carreau-Yasuda nanofluid with thermal radiation and second-order slip," Journal of Thermal Analysis and Calorimetry, vol. 140, no. 3, pp. 1293-1306, 2020.

[9] C. H. Amanulla, S. Saleem, A. Wakif, and M. M. AlQarni, "MHD Prandtl fluid flow past an isothermal permeable sphere with slip effects," Case Studies in Thermal Engineering, vol. 14, Article ID 100447, 2019.

[10] T. Muhammad, H. Waqas, S. A. Khan, R. Ellahi, and S. M. Sait, "Significance of nonlinear thermal radiation in 3D Eyring-Powell nanofluid flow with Arrhenius activation energy," Journal of Thermal Analysis and Calorimetry, pp. 1-16, 2020.

[11] T. Hayat, T. Muhammad, B. Ahmad, and S. A. Shehzad, "Impact of magnetic field in three-dimensional flow of Sisko nanofluid with convective condition," Journal of Magnetism and Magnetic Materials, vol. 413, pp. 1-8, 2016.

[12] A. W. Sisko, "The flow of lubricating greases," Industrial \& Engineering Chemistry, vol. 50, no. 12, pp. 1789-1792, 1958.

[13] J. Buongiorno, "Convective transport in nanofluids," Journal of Heat Transfer, vol. 128, no. 3, pp. 240-250, 2006.

[14] D. A. Nield and A. V. Kuznetsov, "The onset of convection in a horizontal nanofluid layer of finite depth: a revised model," International Journal of Heat and Mass Transfer, vol. 77, pp. 915-918, 2014.

[15] D. A. Nield and A. V. Kuznetsov, "Thermal instability in a porous medium layer saturated by a nanofluid: a revised model," International Journal of Heat and Mass Transfer, vol. 68, pp. 211-214, 2014.

[16] T. Hayat, I. Ullah, T. Muhammad, and A. Alsaedi, "Hydromagnetic squeezed flow of second-grade nanomaterials between two parallel disks," Journal of Thermal Analysis and Calorimetry, vol. 139, no. 3, pp. 2067-2077, 2020.

[17] M. Sajid, M. Mughees, N. Ali, and H. Shahzad, "A theoretical analysis of blade coating for third-grade fluid," Journal of Plastic Film \& Sheeting, vol. 35, no. 3, pp. 218-238, 2019.

[18] S. M. Arifuzzaman, M. S. Khan, A. Al-Mamun, S. Reza-ERabbi, P. Biswas, and I. Karim, "Hydrodynamic stability and heat and mass transfer flow analysis of MHD radiative fourthgrade fluid through porous plate with chemical reaction," Journal of King Saud University-Science, vol. 31, no. 4, pp. 1388-1398, 2019.

[19] J. G. Oldroyd and A. H. Wilson, "On the formulation of rheological equations of state," Proceedings of the Royal Society of London. Series A. Mathematical and Physical Sciences, vol. 200, no. 1063, pp. 523-541, 1950.

[20] R. M. Christensen, "Viscoelastic stress-strain constitutive relations," in Theory Viscoelasticity, pp. 1-34, Academic Press, Cambridge, MA, USA, 2nd edition, 1982.

[21] G. B. Jeffery and L. N. G. Filon, "The motion of ellipsoidal particles immersed in a viscous fluid," Proceedings of the Royal Society of London. Series A, Containing Papers of a Mathematical and Physical Character, vol. 102, no. 715, pp. 161-179, 1922.

[22] T. Hayat, R. S. Saif, R. Ellahi, T. Muhammad, and A. Alsaedi, "Simultaneous effects of melting heat and internal heat generation in stagnation point flow of Jeffrey fluid towards a nonlinear stretching surface with variable thickness,"
International Journal of Thermal Sciences, vol. 132, pp. 344354, 2018.

[23] T. Hayat, T. Muhammad, S. A. Shehzad, and A. Alsaedi, "An analytical solution for magnetohydrodynamic Oldroyd-B nanofluid flow induced by a stretching sheet with heat generation/absorption," International Journal of Thermal Sciences, vol. 111, pp. 274-288, 2017.

[24] G. Koteswara Reddy, K. Yarrakula, C. S. K. Raju, and A. Rahbari, "Mixed convection analysis of variable heat source/sink on MHD Maxwell, Jeffrey, and Oldroyd-B nanofluids over a cone with convective conditions using Buongiorno's model," Journal of Thermal Analysis and Calorimetry, vol. 132, no. 3, pp. 1995-2002, 2018.

[25] A. Awias, K. Masood, and A. Jawad, "Mixed convective flow of Maxwell nanofluid induced by vertically rotating cylinder," Applied Nanoscience, pp. 1-12, 2020.

[26] J. M. Burgers, "Mechanical considerations-model systemsphenomenological theories of relaxation and of viscosity," First report on viscosity and plasticity, Nordemann Publishing Company, New York, NY, USA, 1935.

[27] T. Hayat, A. Aziz, T. Muhammad, and A. Alsaedi, "On model for flow of Burgers nanofluid with Cattaneo-Christov double diffusion," Chinese Journal of Physics, vol. 55, no. 3, pp. 916-929, 2017.

[28] N. Casson, "A flow equation for pigment-oil suspensions of the printing ink type," in Rheology of Dispersed Systems, C. C. Mill, Ed., pp. 84-104, Pergamon Press, Oxford, UK, 1959.

[29] V. C. Kelessidis and R. Maglione, "Modeling rheological behavior of bentonite suspensions as Casson and RobertsonStiff fluids using Newtonian and true shear rates in Couette viscometry," Powder Technology, vol. 168, no. 3, pp. 134-147, 2006.

[30] R. E. Robertson and H. A. Stiff, "An improved mathematical model for relating shear stress to shear rate in drilling fluids and cement slurries," Society of Petroleum Engineers Journal, vol. 16, no. 1, pp. 31-36, 1976.

[31] M. Qasim and S. Noreen, "Heat transfer in the boundary layer flow of a Casson fluid over a permeable shrinking sheet with viscous dissipation," The European Physical Journal Plus, vol. 129, no. 1, pp. 1-8, 2014.

[32] M. M. Bhatti, S. Ullah Khan, O. Anwar Bég, and A. Kadir, "Differential transform solution for hall and ion-slip effects on radiative-convective Casson flow from a stretching sheet with convective heating," Heat Transfer, vol. 49, no. 2, pp. 872-888, 2020.

[33] S. Rosseland, Astrophysik und Atom-Theoretische Grundlagen, Springer-Verlag, Berlin, Germany, 1931.

[34] S. Noreen, A. Malik, and M. M. Rashidi, "Peristaltic flow of shear thinning fluid via temperature-dependent viscosity and thermal conductivity," Communications in Theoretical Physics, vol. 71, no. 4, pp. 367-376, 2019.

[35] R. E. Bellman and R. E. Kalaba, Quasilinearization and Nonlinear Boundary-Value Problems, Elsevier, New York, NY, USA, 1965.

[36] M. Afridi, A. Wakif, M. Qasim, and A. Hussanan, "Irreversibility analysis of dissipative fluid flow over A curved surface stimulated by variable thermal conductivity and uniform magnetic field: utilization of generalized differential quadrature method," Entropy, vol. 20, no. 12, pp. 943-1015, 2018.

[37] M. Qasim, Z. Ali, A. Wakif, and Z. Boulahia, "Numerical simulation of MHD peristaltic flow with variable electrical conductivity and joule dissipation using generalized 
differential quadrature method," Communications in Theoretical Physics, vol. 71, no. 5, pp. 509-518, 2019.

[38] M. Qasim, M. I. Afridi, A. Wakif, and S. Saleem, "Influence of variable transport properties on nonlinear radioactive Jeffrey fluid flow over a disk: utilization of generalized differential quadrature method," Arabian Journal for Science and Engineering, vol. 44, no. 6, pp. 5987-5996, 2019.

[39] M. Qasim, M. I. Afridi, A. Wakif, T. N. Thoi, and A. Hussanan, "Second law analysis of unsteady MHD viscous flow over a horizontal stretching sheet heated non-uniformly in the presence of ohmic heating: utilization of gear-generalized differential quadrature method," Entropy, vol. 21, no. 3, p. $240,2019$.

[40] A. Wakif, M. Qasim, M. I. Afridi, S. Saleem, and M. M. AlQarni, "Numerical examination of the entropic energy harvesting in a magnetohydrodynamic dissipative flow of Stokes' second problem: utilization of the gear-generalized differential quadrature method," Journal of Non-Equilibrium Thermodynamics, vol. 44, no. 4, pp. 385-403, 2019.

[41] M. K. Nayak, A. Wakif, I. L. Animasaun, and M. S. H. Alaoui, "Numerical differential quadrature examination of steady mixed convection nanofluid flows over an isothermal thin needle conveying metallic and metallic oxide nanomaterials: a comparative investigation," Arabian Journal for Science and Engineering, 2020.

[42] C. Shu, Differential Quadrature and its Application in Engineering, Springer Science \& Business Media, Berlin, Germany, 2012.

[43] T. Fang, J. Zhang, and Y. Zhong, "Boundary layer flow over a stretching sheet with variable thickness," Applied Mathematics and Computation, vol. 218, no. 13, pp. 7241-7252, 2012.

[44] C. Y. Wang, "Free convection on a vertical stretching surface," ZAMM-Journal of Applied Mathematics and Mechanics/ Zeitschrift für Angewandte Mathematik und Mechanik, vol. 69, no. 11, pp. 418-420, 1989.

[45] F. Mabood, W. A. Khan, and A. I. M. Ismail, "MHD boundary layer flow and heat transfer of nanofluids over a nonlinear stretching sheet: a numerical study," Journal of Magnetism and Magnetic Materials, vol. 374, pp. 569-576, 2015.

[46] S. S. Motsa, "A new spectral local linearization method for nonlinear boundary layer flow problems," Journal of Applied Mathematics, vol. 2013, Article ID 423628, 15 pages, 2013.

[47] N. A. Shah, I. L. Animasaun, R. O. Ibraheem, H. A. Babatunde, N. Sandeep, and I. Pop, "Scrutinization of the effects of Grashof number on the flow of different fluids driven by convection over various surfaces," Journal of Molecular Liquids, vol. 249, pp. 980-990, 2018.

[48] I. L. Animasaun, R. O. Ibraheem, B. Mahanthesh, and H. A. Babatunde, "A meta-analysis on the effects of haphazard motion of tiny/nano-sized particles on the dynamics and other physical properties of some fluids," Chinese Journal of Physics, vol. 60, pp. 676-687, 2019.

[49] A. Wakif, I. L. Animasau, P. V. Satya Narayana, and G. Sarojamma, "Meta-analysis on thermo-migration of tiny/ nano-sized particles in the motion of various fluids," Chinese Journal of Physics, 2019. 